Homology, Homotopy and Applications, vol.3, No.7, 2001, pp.139-163

\title{
HOMOTOPY RINEHART COHOMOLOGY OF HOMOTOPY LIE-RINEHART PAIRS
}

\author{
LARS KJESETH
}

(communicated by Larry Lambe)

\begin{abstract}
We define homotopy Lie-Rinehart pairs and the associated homotopy Rinehart algebra in the context of coalgebras. We define homotopy Lie-Rinehart resolutions and present conditions under which the associated homotopy Rinehart algebra is a cohomological model for the Rinehart algebra of the resolved Lie-Rinehart pair.
\end{abstract}

\section{Introduction}

The Rinehart cohomology of homotopy Lie-Rinehart resolutions of Lie-Rinehart pairs arose from the study of the BFV (Batalin, Fradkin and Vilkovisky, [FV75], [BV77], [BF83], [BV83] and [BV85]) formulation of classical BRST cohomology (Becchi, Rouet and Stora [BRS75] and, independently, Tyutin [Tyu75]). The classical BRST algebra $(\mathcal{A}, \mathcal{D})$ (see, for example, [Kim92b], [Kim93], [Sta92], [Kim92a], [FHST89], [Sta88], [Sta96], [KS87] and [HT92]) is a differential graded Poisson algebra which, in certain cases, is a cohomological model for the Rinehart algebra $\left(A l t_{\mathbf{B}}(\mathfrak{g}, \mathbf{B}), \delta_{R}\right)$ of alternating $\mathbf{B}$-multilinear functions from a Lie algebra $\mathfrak{g}$ into the associative algebra $\mathbf{B}$ [Rin63]. The Rinehart complex is a subcomplex of the Chevalley-Eilenberg complex $([\mathbf{C E} 48])$ precisely when $(\mathbf{B}, \mathfrak{g})$ is a Lie-Rinehart pair satisfying certain Lie-Rinehart relations (see below).

In homological algebra, a traditional strategy is to replace both $\mathbf{B}$ and $\mathfrak{g}$ in $A l t_{\mathbf{B}}(\mathfrak{g}, \mathbf{B})$ with free or projective resolutions $\mathbf{R}_{\mathbf{B}}$ and $\mathbf{R}_{\mathfrak{g}}$ which have the same or similar algebraic structures, at least up to homotopy. The new object $A l t_{\mathbf{R}_{\mathbf{B}}}\left(\mathbf{R}_{\mathfrak{g}}, \mathbf{R}_{\mathbf{B}}\right)$ is a cohomological model for $\operatorname{Alt}_{\mathbf{B}}(\mathfrak{g}, \mathbf{B})$ if $\operatorname{Alt}_{\mathbf{R}_{\mathbf{B}}}\left(\mathbf{R}_{\mathfrak{g}}, \mathbf{R}_{\mathbf{B}}\right)$ is a differential graded commutative algebra (a dcga) with the same cohomology as $A l t_{\mathbf{B}}(\mathfrak{g}, \mathbf{B})$. In contrast, the BFV construction of the classical BRST algebra (see, for example, [Kim93]) begins by replacing $\mathbf{B}$ with the Koszul-Tate resolution [Tat57], but side-steps replacing $\mathfrak{g}$ with a suitable resolution, opting instead to adjoin formal (ghost) variables to the Koszul-Tate resolution. They then exploit a graded Poisson bracket to construct a differential. Attempting to construct the dcga $A l t_{\mathbf{R}_{\mathbf{B}}}\left(\mathbf{R}_{\mathfrak{g}}, \mathbf{R}_{\mathbf{B}}\right)$ fails if we resolve $\mathbf{B}$ and $\mathfrak{g}$ without preserving (as far as possible) the Lie-Rinehart structure of the pair $(\mathbf{B}, \mathfrak{g})$. Our view in this paper is that a model for a Rinehart complex should

Received January 8, 2001, revised May 3, 2001; published on June 21, 2001.

2000 Mathematics Subject Classification: primary 17B55, 17B81; secondary 17B56, 17 B99.

Key words and phrases: strongly homotopy Lie algebras, Lie-Rinehart pairs, Rinehart cohomology, BRST cohomology.

(C) 2001, Lars Kjeseth. Permission to copy for private use granted. 
be Rinehart-like. But to resolve a Lie-Rinehart pair with a new pair $\left(\mathbf{R}_{\mathbf{B}}, \mathbf{R}_{\mathfrak{g}}\right)$ which is Lie-Rinehart, we must give up a strictly Lie structure on $\mathbf{R}_{\mathfrak{g}}$ in favor of a strongly homotopy Lie structure.

In its earliest incarnation (see [Sul77] and [SS]) a strongly homotopy Lie (shLie) algebra was defined on the tensor coalgebra $\mathrm{T}^{c} L$ of a graded module $L$. In [LM95] and [LS93], the shLie structure was shifted (almost by brute force) onto the graded symmetric coalgebra on the suspension of $L$, that is, on $\bigwedge(s L)$. This transition seemed inelegant and we felt there must be smoother means and more satisfying motivations for making the move. By taking a close look at choices of grading and specific actions of the symmetric group on a graded vector space, we discovered we could identify a subcoalgebra $₫ L$ of $T^{c} L$, which is isomorphic to $\bigwedge(s L)$ and on which an shLie structure can be defined as it was on $\mathrm{T}^{c} L$ in [LM95] and [LS93].

\section{Grading and Sign Conventions}

In order to understand the language of this paper, we must first establish our grading and sign conventions.

All vector spaces, algebras and coalgebras in this paper are over a field $k$ of characteristic zero. All tensor products are over $k$. All maps are at least $k$-linear or $k$-multilinear.

Let $V=\left\{V_{p}\right\}_{0}^{\infty}$ be a graded vector space over $k$ or, more generally, a graded module over an algebra $A$ over $k$. There are four distinct gradings on the tensor module $\otimes V=\left\{V^{\otimes n}\right\}_{n=0}^{\infty}$ : the external, internal, combined and total gradings. We shall denote $v_{1} \otimes \cdots \otimes v_{n} \in V^{\otimes n}$ by $v_{[1 \text { to } n]}$. The external degree, $\operatorname{ed}\left(v_{[1 \text { to } n]}\right)$ is given by the number $n$ of tensor components. The internal degree, $\mathbf{i d}\left(v_{[1}\right.$ to $\left.\left.n\right]\right)$ comes from the internal grading on $V$; each tensor component $v_{i}$ is an element of $V_{p_{i}}$ for some $p_{i}$. The internal degree is the sum $\sum_{i=1}^{n} p_{i}$. The combined degree $\left\|v_{\left[\begin{array}{ll}1 & \\ \text { to }\end{array}\right]}\right\|$ is the vector $\left\langle\operatorname{ed}\left(v_{[1 \text { to } n]}\right), \mathbf{i d}\left(v_{[1}\right.\right.$ to $\left.\left.\left.n\right]\right)\right\rangle$. Finally, for any bigraded object, the total degree $\operatorname{td}\left(v_{[1 \text { to } n]}\right)$ is the $\operatorname{sum} \operatorname{ed}\left(v_{[1 \text { to } n]}\right)+\mathbf{i d}\left(v_{[1 \text { to } n]}\right)$.

A function $f: \otimes V \rightarrow \otimes V$ is of homogeneous degree $r$ with respect to a specific grading if $f\left((\otimes V)_{p}\right) \subset(\otimes V)_{p+r}$ for all $p$. For each of the four gradings, the degree of the homogeneous map $f$ is the difference between the degree of $v_{[1 \text { to } n]} \in \otimes V$ and the degree of its non-zero image under $f$.

The Koszul sign convention states that exchanging two objects of homogeneous degrees $p$ and $q$ (whether elements or maps) introduces a factor of $(-1)^{p q}$. In this paper, we apply the Koszul sign convention consistently, no matter which grading. In the case of the combined grading, the product of $p$ and $q$ will be the dot product of the degree vectors.

Since an shLie algebra $\mathbf{R}_{\mathfrak{g}}$ which resolves a Lie algebra $\mathfrak{g}$ is defined on a graded coalgebra, it is convenient to realize the Lie algebra $\mathfrak{g}$ on a graded colagebra as well. To do this, we must decide what degree to assign to each element of the Lie algebra. If each element of $\mathfrak{g}$ is assigned an external degree of one and an internal degree of zero, we have essentially three choices for describing $\mathfrak{g}$ as a coalgebra. In each case, the skew-commutativity of $l_{2}$ appears as the result of applying the Koszul sign convention. If we select the natural bigrading on $\mathrm{T}^{c} \mathfrak{g}$, the element $x$ has bidegree 
$\langle 1,0\rangle$, which we treat as a vector, and

$$
l_{2}(x \otimes y)=(-1)^{\langle 1,0\rangle \cdot\langle 1,0\rangle} l_{2}(y \otimes x)=-l_{2}(y \otimes x) .
$$

We might instead choose the total degree, in which case the element $x$ has degree $0+1=1$. Here again,

$$
l_{2}(x \otimes y)=(-1)^{1 \cdot 1} l_{2}(y \otimes x)=-l_{2}(y \otimes x) .
$$

Finally, if we consider the tensor coalgebra on the suspension of $\mathfrak{g}$ under the internal grading, then the element $x$ again has degree 1 and $l_{2}$ again appears skewcommutative, but is actually graded commutative.

When it resolves a Lie algebra $\mathfrak{g}$, an shLie algebra $\mathbf{R}_{\mathfrak{g}}$ must operate as a differential object, i.e., its differential must have degree -1 with respect to a single grading. So the shLie algebra resolution $\mathbf{R}_{\mathfrak{g}}$ of $\mathfrak{g}$ must be graded either with respect to the total degree or with respect to the suspended internal degree. Since the differential graded commutative (dgc) algebra $\mathbf{R}_{\mathbf{B}}$ which resolves $\mathbf{B}$ is graded by internal degree, the suspended internal grading for the shLie algebra resolution is the preferred choice. Therefore, we will also view the Lie algebra $\mathfrak{g}$ in its suspended form $s \mathfrak{g}$.

\section{Background}

For any smooth manifold $M$, the set of smooth functions, $C^{\infty}(M)$, is an associative commutative algebra and the set of smooth vector fields on the manifold, $\Gamma(T M)$, forms a Lie algebra. Both $C^{\infty}(M)$ and $\Gamma(T M)$ are modules over each other. Moreover, the the module actions satisfy the equations $(f X) \cdot g=f(X \cdot g)$ and $[X, f Y]=f[X, Y]+(X \cdot f) Y$ for all $f, g \in C^{\infty}(M)$ and $X, Y \in \Gamma(T M)$. A Lie-Rinehart pair is a couple $(\mathbf{B}, \mathfrak{g})$ which admits the analogous structure, where $\mathfrak{g}$ is a Lie algebra (we will suspend it later), $\mathbf{B}$ is an algebra, and both are modules over each other.

Definition 1.1. [Rin63] We denote the left $\mathbf{B}$-module action $\mu$ on $\mathfrak{g}$ by $\mu(a \otimes \alpha):=$

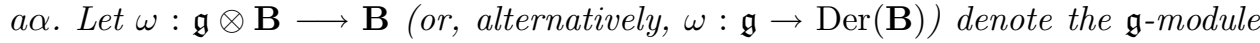
action on $\mathbf{B}$. The pair $(\mathbf{B}, \mathfrak{g})$ is a Lie-Rinehart pair, provided the Lie-Rinehart relations (LRa) and (LRb) are satisfied for all $a, b \in \mathbf{B}$ and $x, y \in \mathfrak{g}$ :

LRa: $\omega(a x \otimes b)=a \cdot \omega(x \otimes b)$, where $\cdot$ indicates the multiplication on $\mathbf{B}$.

LRb: $[x, a y]=a[x, y]+\omega(x \otimes a) y$.

The term Lie-Rinehart pair is not widely used. More often, $\mathfrak{g}$ has been called a $(\mathbf{B}, k)$-Lie algebra, [Rin63] [Pal61] and [Her72]. More recently, Lie-Rinehart pairs have appeared as Lie algebroids (see $[\mathbf{d S W}]$ ).

We will suppose that $\mathfrak{g}$ is finitely generated over $\mathbf{B}$, that is to say, with respect to a generating set $\left\{X_{\alpha}\right\}$, every element $x$ of $\mathfrak{g}$ can be expressed as the sum $b^{\alpha} X_{\alpha}$ where $b^{\alpha} \in \mathbf{B}$. (We use the Einstein summation convention throughout.) The first Lie-Rinehart relation (LRa) states that $\omega$ is $\mathbf{B}$-linear in $\mathfrak{g}$. We can use the second Lie-Rinehart relation ( $\mathrm{LRb}$ ) and its skew-symmetric counterpart to show that the bracket must have the form

$$
\left[b^{\alpha} X_{\alpha}, b^{\beta} X_{\beta}\right]=b^{\alpha} b^{\beta}\left[X_{\alpha}, X_{\beta}\right]+\omega\left(b^{\alpha} X_{\alpha} \otimes b^{\beta}\right) X_{\beta}-\omega\left(b^{\beta} X_{\beta} \otimes b^{\alpha}\right) X_{\alpha}
$$


for any element $b^{\alpha} X_{\alpha} \otimes b^{\beta} X_{\beta}$ of $\mathfrak{g} \otimes \mathfrak{g}$. In the rest of the paper, we will replace the second Lie-Rinehart relation (LRb) in 1 with this equivalent, but more symmetric form.

For any Lie algebra $\mathfrak{g}$ and $\mathfrak{g}$-module $\mathbf{B}$, an $n$-multilinear function $f_{n}: \mathfrak{g}^{\times n} \rightarrow \mathbf{B}$ is alternating if

$$
f_{n}\left(x_{1}, \ldots, x_{i}, x_{i+1}, \ldots, x_{n}\right)=-f_{n}\left(x_{1}, \ldots, x_{i+1}, x_{i}, \ldots, x_{n}\right) .
$$

The Chevalley-Eilenberg complex is the set of all alternating multilinear functions $A l t_{k}(\mathfrak{g}, \mathbf{B})$, graded by $n$, and equipped with a degree +1 differential $\delta_{C E}$ : $A l t_{k}^{n}(\mathfrak{g}, \mathbf{B}) \rightarrow A l t_{k}^{n+1}(\mathfrak{g}, \mathbf{B})$ given by

$$
\begin{aligned}
\delta_{\mathrm{CE}} f_{n}\left(x_{0}, \ldots, x_{n}\right)=\sum_{i=0}^{n}(-1)^{i} \omega\left(x_{i} \otimes f_{n}\left(x_{0}, \ldots, \widehat{x}_{i}, \ldots, x_{n}\right)\right. & \\
& \quad-\sum_{i<j}(-1)^{i+j-1} f_{n}\left(\left[x_{i}, x_{j}\right], x_{0}, \ldots, \widehat{x}_{i}, \ldots, \widehat{x}_{j}, \ldots, x_{\sigma(n+1)}\right),
\end{aligned}
$$

where $\widehat{x_{k}}$ indicates that $x_{k}$ should be omitted. The image under $\delta_{C E}$ of any element $b \in \mathbf{B} \approx A l t_{k}^{0}(\mathfrak{g}, \mathbf{B})$ is defined by setting $\delta_{C E} b(x)=\omega(x \otimes b)$. When $\mathbf{B}$ is an algebra, the Chevalley-Eilenberg complex is a differential graded commutative algebra. For $f_{n}$ and $g_{m}$ in $\operatorname{Alt}_{k}(\mathfrak{g}, \mathbf{B})$, the product $f_{n} \smile g_{m}$ is given by

$$
\left(f_{n} \smile g_{m}\right)\left(x_{1}, \ldots, x_{n+m}\right)=\sum_{\substack{\sigma \\(n, m) \\ \text { unshuffles }}} f_{n}\left(x_{\sigma(1)}, \ldots, x_{\sigma(n)}\right) g_{m}\left(x_{\sigma(n+1)}, \ldots, x_{\sigma(n+m)}\right) .
$$

An $(n, m)$ - unshuffle is any permutation $\sigma$ in the symmetric group $\Sigma_{n+m}$ such that

$$
\underbrace{\sigma(1)<\cdots<\sigma(n)}_{\text {first } \sigma \text { hand }} \quad \text { and } \quad \underbrace{\sigma(n+1)<\cdots<\sigma(n+m),}_{\text {second } \sigma \text { hand }}
$$

where $\sigma(j)$ is the element of the set $\{1, \ldots, n+m\}$ moved to the $j^{\text {th }}$ position under $\sigma$. The differential $\delta_{C E}$ acts as a derivation with respect to this multiplication. The cohomology of this complex with respect to $\delta_{C E}$ is the Chevalley-Eilenberg cohomology of $\mathfrak{g}$ with coefficients in B [CE48].

Suppose $(\mathbf{B}, \mathfrak{g})$ is a Lie-Rinehart pair and the alternating function $f_{n}$ is $\mathbf{B}$ multilinear, i.e., $f_{n}\left(a_{1} x_{1}, \ldots, a_{n} x_{n}\right)=a_{1} \cdots a_{n} f_{n}\left(x_{1}, \ldots, x_{n}\right)$. Because the Lie action map $\omega$ maps $\mathfrak{g}$ into the derivations of $\mathbf{B}$ and as a result of the Lie-Rinehart relations, the image of $f_{n}$ under the Chevalley-Eilenberg differential $\delta_{C E}$ is again $\mathbf{B}$-multilinear, despite the fact that the bracket is not $\mathbf{B}$-multilinear. The Rinehart algebra $R=A l t_{\mathbf{B}}(\mathfrak{g}, \mathbf{B})$ with differential $\delta_{R}=\delta_{C E}$ is a subcomplex of the Chevalley-Eilenberg algebra and the cohomology with respect to $\delta_{R}$ is the Rinehart cohomology of $\mathfrak{g}$ with coefficients in B [Rin63].

\section{Summary}

First, we define the Lie algebras of subordinate derivation sources, resting coderivations and shared Lie modules (§2). Following the lead of [LM95] and [LS93], we 
define both homotopy and non-homotopy Lie algebras, Lie algebra modules, LieRinehart pairs and Rinehart cohomology, all in the coalgebra setting ( $\S \S 3$ and 4). We piece together homotopy Lie-Rinehart resolutions for Lie-Rinehart pairs and present conditions under which the homotopy Rinehart algebra for a homotopy Lie-Rinehart resolution is a model for the Rinehart cohomology complex for a LieRinehart pair $(\$ 5)$.

We have omitted all sign arguments from the proofs in this paper, as they are not generally instructive.

\section{Subordinate derivation sources, resting coderivations and shared Lie modules}

\section{Motivation}

The following brief and not unduly precise reexamination of the Lie-Rinehart relations and the Rinehart differential provides motivation for why coalgebras are an appropriate setting for both the homotopy and the non-homotopy versions of Lie algebras, Lie modules, Chevalley-Eilenberg cohomology and Rinehart cohomology. In particular, the discussion below highlights why we define subordinate and resting coderivations, as well as shared Lie modules.

The underlying module structure for the tensor coalgebra $T^{c} \mathfrak{g}$ on $\mathfrak{g}$ is $\bigotimes \mathfrak{g}$, which is the collection $\left\{\mathfrak{g}^{\otimes n}\right\}_{n=0}^{\infty}$.

With malice aforethought, we rename the bracket as $l_{2}$ and extend $l_{2}$ to a map from $\mathfrak{g}^{\otimes n} \rightarrow \mathfrak{g}^{\otimes n-1}$ for any $n>2$ by setting (up to sign)

$$
l_{2}\left(x_{[1 \text { to } n]}\right)=\sum_{i=1}^{n-1} \pm\left(1^{\otimes i-1} \otimes l_{2} \otimes 1^{\otimes n-(i+1)}\right)\left(x_{[1 \text { to } n]}\right),
$$

where $\left(1^{\otimes i-1} \otimes l_{2} \otimes 1^{\otimes n-(i+1)}\right)\left(x_{[1}\right.$ to $\left.\left.n\right]\right)=$

$$
\pm x_{[1 \text { to } i-1]} \otimes l_{2}\left(x_{i} \otimes x_{i+1}\right) \otimes x_{[i+2 \text { to } n]} .
$$

The bracket $l_{2}$ is now a coderivation on $\mathrm{T}^{c} \mathfrak{g}([\mathbf{L a d} \mathbf{9 9}]$ and $[\mathbf{G L 8 9}])$, although it has lost its skew-commutativity. We regain the symmetry of the bracket by passing to an appropriate subcoalgebra $\mathbb{Q g}$, which we define below. We will denote elements

of this coalgebra by $x_{1} \otimes \cdots \otimes x_{n}$ or $x_{[1 \text { to } n]}^{\otimes}$. In this context, the Jacobi identity is the fact that $l_{2} \circ l_{2}=0$. Or, since the set of coderivations on a coalgebra forms a Lie algebra, the Jacobi identity is equivalent to $\left[l_{2}, l_{2}\right]=0$.

Similarly, we rename the Lie action map $\omega$ as $m_{2}$ and extend $m_{2}$ as a coderivation on $\triangle \mathfrak{g} \otimes \mathbf{B}$ which is subordinate to $l_{2}$ by setting $m_{2}\left(x_{[1 \text { to } n]}^{\otimes} \otimes b\right)=$

$$
l_{2}\left(x_{[1 \text { to } n]}^{\otimes}\right) \otimes b \quad \pm \sum_{\substack{\sigma \\(n-1,1)}} x_{\sigma[1 \text { to } n-1]}^{\otimes} \otimes m_{2}\left(x_{\sigma(n)} \otimes b\right)
$$

for any $n>1$. The symbol $x_{\sigma[1 \text { to } n]}^{\bigotimes}$ is shorthand for $x_{\sigma(1)} \otimes \cdots \otimes x_{\sigma(n)}$, where $\sigma(i)$ is the element of the ordered set $\{1, \ldots, k\}$ which moves to the $i^{\text {th }}$ position 
under the $(n-1,1)$-unshuffle $\sigma$. The condition any Lie action must satisfy becomes $m_{2} \circ m_{2}=0$ or $\left[m_{2}, m_{2}\right]=0$.

For the Lie-Rinehart pair $(\mathbf{B}, \mathfrak{g})$, the Lie algebra is a module over $\mathbf{B}$, but the bracket $l_{2}$ is not $\mathbf{B}$-linear. Rather, the second Lie-Rinehart relation states that $l_{2}(a x \otimes b y)$ consists of a B-linear piece, $a b l_{2}(x \otimes y)$, and two other terms, $m_{2}(a x \otimes$ b) $y-m_{2}(b y \otimes a) x$ (compare with equation 1). Any coderivation which shares this property with a specific subordinate coderivation is called a resting coderivation. In particular, $l_{2}$ rests on $m_{2}$.

Finally, any alternating function $f: \mathfrak{g}^{\otimes n} \rightarrow \mathbf{B}$ is a linear function on $\mathfrak{g} \otimes^{n}$. We extend $f_{n}$ to a function from $\ \mathfrak{g}$ into $\triangle \mathfrak{g} \otimes \mathbf{B}$ by setting $f_{n}\left(x_{[1 \text { to } k+n]}^{\otimes}\right)=$

$$
\sum_{\substack{\sigma \\(k, n)}} \pm x_{\sigma[1 \text { to } k]}^{\bigotimes} \otimes f_{n}\left(x_{\sigma[k+1 \text { to } k+n]}^{\otimes}\right)
$$

on $\mathfrak{g} \otimes k+n$ and setting $f_{n}=0$ on $\mathfrak{g} \otimes^{i}$ for $i<n$. Using equations (3), (4) and (5), the Chevalley-Eilenberg differential (and hence, the Rinehart differential) becomes

$$
\delta_{C E} f_{n}=m_{2} \circ f_{n} \pm f_{n} \circ l_{2},
$$

which looks remarkably like some sort of commutator bracket action, whence the definiton of a shared Lie module given below.

In our more rigorous discussion of these ideas, we focus on the following algebraic objects.

1. The graded Lie algebra $\operatorname{Coder}_{W}^{W}(\bigwedge(s V))$ is the set of all coderivations on the free graded commutative algebra $\Lambda(s V)$, each of which rests on its own specific subordinate coderivation in the Lie algebra $\operatorname{Coder}_{W}^{W}(\bigwedge(s V) \otimes W)$ (see 2).

2. For a graded commutative algebra $W$, the Lie algebra $\operatorname{Coder}_{W}^{W}(\bigwedge(s V) \otimes W)$ is the set of all coderivations, each of which is subordinate to a specific resting coderivation and is also a $W$-derivation source. A $W$-derivation source $m$ is the extension as a coderivation onto $\bigwedge(s V)$ of a map $m$ on $(s V)^{\wedge n} \otimes W$ which acts like a Lie module structure map, i.e., $m:(s V)^{\wedge n} \rightarrow \operatorname{Der}(W)$.

3. The graded commutative algebra $\operatorname{Hom}_{W}(\bigwedge(s V), \bigwedge(s V) \otimes W)$ is the set of all $W$-linear maps on $\bigwedge(s V)$ with coefficients in $\bigwedge(s V) \otimes W$ and it admits a shared Lie module structure over both $\operatorname{Coder}_{W}^{W}(\bigwedge(s V) \otimes W)$ and $\operatorname{Coder}_{W}^{W}(\bigwedge(s V))$.

We begin our more formal treatment with a return to the basics.

\section{Coalgebras and Subcoalgebras}

A graded coassociative coalgebra is a pair $(\mathbf{C}, \Delta)$, where $\mathbf{C}$ is a graded module over $k$ together with a 0-degree coassociative comultiplication $\Delta: \mathbf{C} \rightarrow \mathbf{C} \otimes \mathbf{C}$. A function $f$ with degree $|f|=r$ is a coderivation on $\mathbf{C}$ if $\Delta f=\left(f \otimes 1_{\mathbf{C}}+1_{\mathbf{C}} \otimes f\right) \Delta$.

The set of all coderivations on a coalgebra $C$, denoted $\operatorname{Coder}(C)$, is a graded Lie algebra under the graded commutator bracket, that is to say, $[f, g]=f g-$ $(-1)^{|f||g|} g f$ for all $f$ and $g \in \operatorname{Coder}(C)$, where $|f|$ and $|g|$ are the degrees of $f$ and $g$. For a graded module $V$, the tensor module $\otimes V=\left\{V^{\otimes n}\right\}$ is a graded coassociative 
coalgebra. We will denote an element of $V^{\otimes n}$ by $v_{[1 \text { to } n]}$. The coalgebra $T^{c} V$ is equipped with the standard comultiplication $\Delta: \mathrm{T}^{c} V \rightarrow \mathrm{T}^{c} V \otimes \mathrm{T}^{c} V$ given by

$$
\Delta\left(v_{[1 \text { to } n]}\right)=\sum_{j=0}^{n}\left(v_{[1 \text { to } j]}\right) \otimes\left(v_{[j+1 \text { to } n]}\right),
$$

where $V^{\otimes 0} \approx k$. The terms with $j=0$ and $j=n$ are $1 \otimes v_{[1 \text { to } n]}$ and $v_{[1 \text { to } n]} \otimes 1$, respectively.

Actions of the symmetric group $\Sigma_{\infty}$ on $\mathrm{T}^{c} V$

An action $\rho_{n}$ of the symmetric group $\Sigma_{n}$ on $V^{\otimes n}$ is any homomorphism

$$
\rho_{n}: \Sigma_{n} \rightarrow \operatorname{End}\left(V^{\otimes n}\right) .
$$

An action $\rho=\left\{\rho_{n}\right\}_{n=1}^{\infty}$ of $\left\{\Sigma_{n}\right\}_{n=1}^{\infty}$ on $\mathrm{T}^{c} V$ is defined in the obvious way. The $\rho$-invariant subspace of $\mathrm{T}^{c} V$ forms a subcoalgebra. The two actions and associated subcoalgebras which play a role in this paper are listed below. The first one is the familiar graded commutative coalgebra $\bigwedge V$. The other one helps place this paper in its historical context.

1. The internal graded action $\rho^{\wedge}$ recognizes only the internal grading on $V^{\otimes n}$ and is given by $\sigma \cdot\left(v_{[1 \text { to } n]}\right)=\kappa_{\text {id }}(\sigma) v_{\sigma[1 \text { to } n]}$ for all $n, \sigma \in \Sigma_{n}$ and $v_{[1 \text { to } n]} \in V^{\otimes n}$. Since $\Sigma_{n}$ acts transitively on itself, the $\rho^{\wedge}$-invariant subspace $\bigwedge V$ in $\mathrm{T}^{c} V$ is the internal graded symmetric coalgebra on $V$ and is generated by elements of the form

$$
\sum_{\sigma \in \Sigma_{n}} \kappa_{\mathbf{i d}}(\sigma) v_{\sigma[1 \text { to } n]}
$$

which we will denote by $v_{[1 \text { to } n]}^{\wedge}$. For all $v_{[1 \text { to } n]}^{\wedge} \in V^{\wedge n}$ and $\sigma \in \Sigma_{n}$, we have the commutation relation $v_{[1 \text { to } n]}^{\wedge}=\kappa_{\mathbf{i d}}(\sigma) v_{\sigma[1 \text { to } n]}^{\wedge}$. The coassociative comultiplication on $\bigwedge V$ is given by

$$
\Delta\left(v_{[1 \text { to } n]}^{\wedge}\right)=\sum_{j=0}^{n} \sum_{\substack{\rho \\(j, n-j)-\text { unshuffle }}} \kappa_{\mathbf{i d}}(\rho)\left(v_{\rho[1 \text { to } j]}^{\wedge}\right) \otimes\left(v_{\rho[j+1 \text { to } n]}^{\wedge}\right) .
$$

2. The combined graded action $\rho \mathbb{Q}$ is given by $\sigma \cdot\left(v_{[1 \text { to } n]}\right)=\kappa_{\|\|}(\sigma) v_{\sigma[1}$ to $\left.n\right]$. The $\rho \otimes$-invariant subcoalgebra is the combined graded symmetric coalgebra $(\mathbb{V}$, generated by elements of the form

$$
\sum_{\sigma \in \Sigma_{n}} \kappa_{\|\|}(\sigma) v_{\sigma[1 \text { to } n]} \text {. }
$$

The commutation relation is $v_{[1 \text { to } n]}^{\bigotimes}=\kappa_{\|\|}(\sigma) v_{\sigma[1 \text { to } n]}^{\bigotimes}$, and the comultiplication is given by

$$
\Delta\left(v_{[1 \text { to } n]}^{\otimes}\right)=\sum_{j=0}^{n} \sum_{\substack{\rho \\(j, n-j)}} \kappa_{\|\|}(\rho)\left(v_{\rho[1 \text { to } j]}^{\otimes}\right) \otimes\left(v_{\rho[j+1 \text { to } n]}^{\otimes}\right),
$$


where it is understood that the second sum is over all $(j, n-j)$-unshuffles.

Let $s V$ be the suspension of $V$, that is to say, $(s V)_{p}=V_{p-1}$ for all $p$. The subcoalgebra $\triangle V$ of the tensor coalgebra $\mathrm{T}^{c} V$ is isomorphic to the subcoalgebra $\bigwedge(s V)$ of $\mathrm{T}^{c}(s V)$. This isomorphism replaces the bigrading on $\mathbb{Q} V$ with its total grading, allowing it to function more easily as a differential object. The coalgebra $\bigwedge(s V)$ is graded by internal degree, which will be the preferred setting when looking at graded maps from $\bigwedge(s V)$ into a differential graded commutative algebra, which are traditionally graded by internal degree.

The coalgebra isomorphism $\mathrm{S}: \mathbb{Q} V \rightarrow \bigwedge(s V)$ is not of homogeneous degree; each map $\mathrm{S}^{n}: V \otimes n \rightarrow(s V)^{\wedge n}$ increases the internal degree of an element by $n$. Exchanging a map $f$ with $\mathrm{S}^{n}$ will produce a sign of $(-1)^{\langle 0, n\rangle \cdot\langle\operatorname{ed}(f), \mathbf{i d}(f)\rangle}=$ $(-1)^{n \cdot \mathbf{i d}(f)}$. The inverse isomorphism of $\mathrm{S}^{n}$ shall be denoted by $\mathrm{S}^{-n}$. The map $\mathrm{S}$ respects the appropriate actions of $\Sigma_{\infty}$.

Although we work with maps on $\mathrm{T}^{c}(s V)$, the definition of action invariant maps applies to maps on $\mathrm{T}^{c} V$ as well. Let $W$ be a graded module.

Definition 2.1. A map of graded modules $\widehat{l_{n}}:(s V)^{\otimes n} \rightarrow W$ is $\rho_{n}$-invariant if, for all $s v_{[1 \text { to } n]}$ and $\sigma \in \Sigma_{n}, \widehat{l_{n}}\left(\sigma \cdot\left(s v_{[1 \text { to } n]}\right)\right)=\widehat{l_{n}}\left(s v_{[1 \text { to } n]}\right)$. Similarly, a map $\widehat{l}: \mathrm{T}^{c}(s V) \rightarrow W$ is $\rho$-invariant if $\widehat{l}\left(\sigma \cdot\left(s v_{[1 \text { to } n]}\right)\right)=\widehat{l}\left(s v_{[1 \text { to } n]}\right)$ for all $n$ and $\sigma \in \Sigma_{n}$.

If $\widehat{l}: \mathrm{T}^{c}(s V) \rightarrow W$ is $\rho$-invariant, then $\widehat{l}$ restricted to the $\rho$-invariant subcoalgebra is well-defined because $\Sigma_{n}$ acts transitively on itself. The maps of greatest interest to us in this paper are either suspended internal graded symmetric $\left(\rho^{\wedge}\right.$-invariant) or combined graded symmetric ( $\rho \otimes$-invariant). The map $\widehat{l_{n}}$ is suspended internal graded symmetric if $\widehat{K_{\mathbf{i d}}}(\sigma) \widehat{l_{n}}\left(s v_{\sigma[1}\right.$ to $\left.\left.n\right]\right)=\widehat{l_{n}}\left(s v_{[1 \text { to } n]}\right)$ and $l_{n}$ is combined graded symmetric if $\kappa_{\|\|}(\sigma) l_{n}\left(v_{\sigma[1 \text { to } n]}\right)=l_{n}\left(v_{[1 \text { to } n]}\right)$ for all $v_{[1 \text { to } n]}$ and $\sigma \in \Sigma_{n}$. We do not lose any information if we restrict the map $\widehat{l_{n}}$ to $(s V)^{\wedge n}$ (or restrict $l_{n}$ to $V \otimes^{n}$ ) because

$$
\begin{aligned}
\widehat{l_{n}}\left(s v_{\left[\begin{array}{ll}
1 & \text { to } n]
\end{array}\right)}\right) & =\widehat{l_{n}}\left(\sum_{\sigma \in \Sigma_{n}} \widehat{\kappa_{\mathbf{i d}}}(\sigma) s v_{\sigma[1 \text { to } n]}\right) \\
& =\sum_{\sigma \in \Sigma_{n}} \widehat{\kappa_{\mathbf{i d}}}(\sigma) \widehat{l_{n}}\left(s v_{\sigma[1 \text { to } n]}\right) \\
& \left.=\sum_{\sigma \in \Sigma_{n}} \widehat{l_{n}}\left(s v_{[1} \text { to } n\right]\right) \\
& \left.=n ! \widehat{l_{n}}\left(s v_{[1} \text { to } n\right]\right) .
\end{aligned}
$$

So whenever we consider collections of maps on $\mathrm{T}^{c} V$ with the same invariance, we pass to that invariant subcoalgebra. The isomorphism $S$ implies that any map $l_{n}$ : $V \otimes n \rightarrow V$ induces a map $\widehat{l_{n}}:(s V)^{\wedge n} \rightarrow(s V)$ by setting $\widehat{l_{n}}=(-1)^{n \cdot \mathbf{i d}\left(l_{n}\right)} \mathrm{S}^{1} l_{n} \mathrm{~S}^{-n}$. This process is invertible, so the set of all maps $l_{n}: V \otimes n \rightarrow V$ is isomorphic to the 
set of all maps $\widehat{l_{n}}:(s V)^{\wedge n} \rightarrow s V$. Therefore, whatever we define and prove in the suspended internal graded setting has its analog in the combined graded setting, e.g., all Lie algebras and graded commutative algebra defined in the remainder of this section.

\section{The graded Lie algebra Coder $(\bigwedge(s V))$}

Following [Lad99] and [GL89], one can extend any map $\widehat{l_{n}}:(s V)^{\otimes n} \rightarrow(s V)$ to a coderivation $\widehat{l_{n}}: \mathrm{T}^{c}(s V) \rightarrow \mathrm{T}^{c}(s V)$ by setting $\widehat{l_{n}}=0$ on $(s V)^{\otimes k}$ for $k<n$ and, for $k \geqslant n$, setting

$$
\left.\widehat{l_{n}}\left(s v_{[1} \text { to } k\right]\right)=\sum_{i=1}^{k-n+1}\left(1^{\otimes i-1} \otimes \widehat{l_{n}} \otimes 1^{\otimes k-n+1-i}\right)\left(s v_{[1 \text { to } k]}\right) .
$$

Here, $\left(1^{\otimes i-1} \otimes \widehat{l_{n}} \otimes 1^{\otimes k-n+1-i}\right)\left(v_{[1 \text { to } k]}\right)=$

$$
\left.\widehat{\mathrm{K}_{\mathbf{i d}}}\left(\widehat{l_{n}} ; s v_{[1 \text { to } i-1]}\right) s v_{[1 \text { to } i-1]} \otimes \widehat{l_{n}}\left(s v_{[i \text { to } i+n-1]}\right) \otimes s v_{[i+n} \text { to } k\right],
$$

where $\widehat{\mathrm{K}_{\mathbf{i d}}}\left(\widehat{l_{n}} ; s v_{[1}\right.$ to $\left.\left.i-1\right]\right)$ is the sign introduced by exchanging $\widehat{l_{n}}$ with $s v_{[1}$ to $\left.i-1\right]$. Extending any $\rho^{\wedge}$-invariant map $\widehat{l_{n}}$ as a coderivation on $\mathrm{T}^{c}(s V)$ does not preserve its invariance. However, we can extend (rather elegantly) the restriction of $\widehat{l_{n}}$ on $(s V)^{\wedge n}$ to a coderivation on the $\rho^{\wedge}$-invariance subcoalgebra $\bigwedge(s V)$ by setting

$$
\widehat{l_{n}}\left(s v_{[1 \text { to } k]}^{\wedge}\right)=\sum_{\substack{\rho \\(n, k-n)}} \widehat{\mathrm{K}_{\mathbf{i d}}}(\rho) \widehat{l_{n}}\left(s v_{\rho[1 \text { to } n]}^{\wedge}\right) \wedge s v_{\rho[n+1 \text { to } k]}^{\wedge} .
$$

The reader is left to verify that extending a map $\widehat{l_{n}}$ as in equation (6) produces a well-defined map on $\bigwedge(s V)$. The restriction of the coderivation $\widehat{l_{n}}$ on $\mathrm{T}^{c}(s V)$ to the $\rho^{\wedge}$-invariant subcoalgebra $\bigwedge(s V)$ is equal to the coderivation in equation 6 . The set of all coderivations $\widehat{l_{n}}$ on $\bigwedge(s V)$ generate the Lie algebra $\operatorname{Coder}(\bigwedge(s V))$.

Next, we examine maps $\widehat{m_{n}}:(s V)^{\wedge n-1} \otimes W \rightarrow W$ (the Lie action map $\widehat{m_{2}}$ is such a map) and $\widehat{f_{n}}:(s V)^{\wedge n} \rightarrow W$ (elements of $A_{l} t_{k}(\mathfrak{g}, \mathbf{B})$ are of this form), where $W$ is a graded commutative algebra. We will denote the degree of any element $w \in W$ by $|w|$. Elements of $W$ have no external degree, at least not with respect to $\bigwedge(s V)$.

\section{Subordinate coderivations and $\operatorname{Coder}(\bigwedge(s V) \otimes W)$}

We begin with maps $\widehat{m_{n}}:(s V)^{\wedge n-1} \otimes W \rightarrow W$. Let $\widehat{l_{n}}:(s V)^{\wedge n} \rightarrow s V$ be any map of degree $\mathbf{i d}\left(\widehat{l_{n}}\right)$ and let $\widehat{l}$ be any coderivation on $\Lambda(s V)$ of degree $\mathbf{i d}(\widehat{l})$.

Definition 2.2. A map $\widehat{m_{n}}:(s V)^{\wedge n-1} \otimes W \rightarrow W$ is an $\widehat{l_{n}}$-subordinate map if the degree of $\widehat{m_{n}}$ and $\widehat{l_{n}}$ agree. $A$ map $\widehat{m}: \bigwedge(s V) \otimes W \rightarrow \bigwedge(s V) \otimes W$ is an $\widehat{l}$-subordinate coderivation if $\mathbf{i d}(\widehat{m})=\mathbf{i d}(\widehat{l})$ and $\left(\Delta \otimes 1_{W}\right) \widehat{m}=\left(\widehat{l} \otimes 1_{\wedge(s V) \otimes W}+1_{\wedge(s V)} \otimes \widehat{m}\right)(\Delta \otimes$ $\left.1_{W}\right)$.

Just as the map $\widehat{l_{n}}$ extends to a coderivation on $\bigwedge(s V)$, so too, the $\widehat{l_{n}}$-subordinate map $\widehat{m_{n}}$ may be extended to an $\widehat{l_{n}}$-subordinate coderivation on $\Lambda(s V) \otimes W$. On 
$(s V)^{\wedge k} \otimes W$, we set $\widehat{m_{n}}=0$ for $k<n-1$ and we set $\widehat{m_{n}}=\widehat{l_{n}} \otimes 1_{W}+1^{\wedge k-n+1} \otimes \widehat{m_{n}}$ for all $k>n-1$.

The set of all subordinate coderivations $\operatorname{Coder}(\bigwedge(s V) \otimes W)$ forms a graded Lie algebra under the graded commutator bracket. The bracket respects subordination, that is to say, if $\widehat{m_{i}}$ and $\widehat{m_{j}}$ are $\widehat{l_{i}}$ and $\widehat{l_{j}}$-subordinate coderivations respectively, then $\left[\widehat{m_{i}}, \widehat{m_{j}}\right]$ is a coderivation subordinate to $\left[\widehat{l_{i}}, \widehat{l_{j}}\right]$. (Of course, the suspension map $\mathrm{S}$ respects subordinate maps and coderivations.)

The graded commutative algebra $\operatorname{Hom}(\bigwedge(s V), \bigwedge(s V) \otimes W)$

We can extend any map $\widehat{f_{n}}:(s V)^{\wedge n} \rightarrow W$ to a function from $\bigwedge(s V)$ into $\bigwedge(s V) \otimes W$ by setting $\widehat{f_{n}}=0$ for $k<n$ and, for $k \geqslant n$, setting $\widehat{f_{n}}\left(s v_{[1 \text { to } k]}^{\wedge}\right)=$

$$
\sum_{\substack{\sigma \\(k-n, n)}} \widehat{\mathrm{K}_{\mathbf{i d}}}(\sigma) \widehat{\mathrm{K}_{\mathbf{i d}}}\left(\widehat{f_{n}} ; s v_{\sigma[1 \text { to } k-n]}^{\wedge}\right) s v_{\sigma[1 \text { to } k-n]}^{\wedge} \otimes \widehat{f_{n}}\left(s v_{\sigma[k-n+1 \text { to } k]}^{\wedge}\right) .
$$

The set of all such maps is $\operatorname{Hom}(\bigwedge(s V), \bigwedge(s V) \otimes W)$.

Suppose $\widehat{m_{p}} \in \operatorname{Coder}(\bigwedge(s V) \otimes W)$ is subordinate to $\widehat{l_{p}} \in \operatorname{Coder}(\bigwedge(s V))$. The map

$$
\left\langle\widehat{m_{p}}, \quad\right\rangle: \operatorname{Hom}(\bigwedge(s V), \bigwedge(s V) \otimes W) \rightarrow \operatorname{Hom}(\bigwedge(s V), \bigwedge(s V) \otimes W),
$$

given by setting

$$
\left\langle\widehat{m_{p}}, \widehat{f_{n}}\right\rangle=\widehat{m_{p}} \widehat{f_{n}}-\widehat{\mathrm{K}_{\mathbf{i d}}}\left(\widehat{m_{p}} ; \widehat{f_{n}}\right) \widehat{f_{n}} \widehat{l_{p}}
$$

on $(s V)^{\wedge n+p-1}$ and extending $\left\langle\widehat{m_{p}}, \widehat{f_{n}}\right\rangle$ to all of $\bigwedge(s V)$ as in equation (7) is welldefined. This map exposes an unusual type of graded Lie module structure on $\operatorname{Hom}(\bigwedge(s V), \bigwedge(s V) \otimes W)$. It is, in a sense, a Lie-module over both $\operatorname{Coder}(\bigwedge(s V) \otimes W)$ and $\operatorname{Coder}(\bigwedge(s V))$ simultaneously in the sense that

$$
\left\langle\left[\widehat{m_{i}}, \widehat{m_{j}}\right],\right\rangle=\left\langle\widehat{m_{i}},\left\langle\widehat{m_{j}}, \quad\right\rangle\right\rangle-\widehat{\kappa_{\mathbf{i d}}}\left(\widehat{m_{i}} ; \widehat{m_{j}}\right)\left\langle\widehat{m_{j}},\left\langle\widehat{m_{i}}, \quad\right\rangle\right\rangle .
$$

We will call such a Lie module shared.

When $(\mathbf{B}, s \mathfrak{g})$ is a Lie-Rinehart pair, the Lie module map $\widehat{m_{2}}: \mathfrak{g} \otimes \mathbf{B} \rightarrow \mathbf{B}$ should be a map from $s \mathfrak{g}$ into $\operatorname{Der}(\mathbf{B})$. With this in mind, we introduce the following notion.

Definition 2.3. An $\widehat{l_{n}}$-subordinate map $\widehat{m_{n}}$ is a $W$-derivation source if for every $s v_{[1 \text {, to } n-1]}^{\wedge}$ in $(s V)^{\wedge n-1}$, the map $\widehat{m}_{n}\left(s v_{[1 \text { to } n-1]}^{\wedge} \otimes(\quad)\right): W \rightarrow W$ is a graded derivation on $W$.

In other words, a $W$-derivation source $\widehat{m_{n}}$ is a map from $(s V)^{\wedge n-1}$ into $\operatorname{Der}(W)$. In practice, we will work with $\widehat{m_{n}}$ as a $\widehat{l_{n}}$-subordinate coderivation on $\bigwedge(s V) \otimes W$, where the characterization of $\widehat{m_{n}}$ as a map into $\operatorname{Der}(W)$ is somewhat obscured. The subset of all $W$-derivation sources in $\operatorname{Coder}(\bigwedge(s V) \otimes W)$ forms a graded Lie subalgebra denoted by $\operatorname{Coder}^{W}(\bigwedge(s V) \otimes W)$.

The subset of all coderivations $\widehat{l_{n}} \in \operatorname{Coder}(\bigwedge(s V))$ which admit a subordinate $W$-derivation source $\widehat{m_{n}}$ forms a graded Lie subalgebra which we will denote by $\operatorname{Coder}^{W}(\bigwedge(s V))$. 
The algebra structure on $W$ extends to a graded commutative algebra structure on $\bigwedge(s V) \otimes W$, which provides $\operatorname{Hom}(\bigwedge(s V), \bigwedge(s V) \otimes W)$ with a cup product. If $\widehat{f_{n}}$ and $\widehat{g_{s}}$ are maps in $\operatorname{Hom}(\bigwedge(s V), \bigwedge(s V) \otimes W)$. Then $\widehat{f_{n}} \smile \widehat{g_{s}}$ is given by setting $\left(\widehat{f_{n}} \smile \widehat{g_{s}}\right)\left(s v_{[1 \text { to } n+s]}^{\wedge}\right)=$

$$
\sum_{\substack{\rho \\(n, s)}} \widehat{K_{\mathbf{i d}}}(\rho) \widehat{K_{\mathbf{i d}}}\left(\widehat{g_{s}} ; s v_{\rho[1 \text { to } n]}^{\wedge} \widehat{f_{n}}\left(s v_{\rho[1 \text { to } n]}^{\wedge}\right) \cdot \widehat{g_{s}}\left(s v_{\rho[n+1 \text { to } n+s]}^{\wedge}\right)\right.
$$

on $(s V)^{\wedge n+s}$, where $\cdot$ represents the the multiplication on $W$ (henceforth, the $\cdot$ will be supressed). The map $\widehat{f_{n}} \smile \widehat{g_{s}}$ is then extended to all of $\Lambda(s V)$ as in equation (7). The map $\left\langle\widehat{m_{p}}, \quad\right\rangle$ acts as a derivation with respect to the cup product, so long as $\widehat{m_{p}}$ is a $W$-derivation source.

\section{$W$-linear maps and resting coderivations}

For a Lie-Rinehart pair $(\mathbf{B}, s \mathfrak{g})$, the Rinehart complex of $\mathbf{B}$-linear alternating maps is a subcomplex of the Chevalley-Eilenberg complex without modifying the differential. This is due to the $\mathbf{B}$-linearity of any $\widehat{f_{n}}$ in $\operatorname{Alt}_{\mathbf{B}}(s \mathfrak{g}, \mathbf{B})$, the Lie-Rinehart relations, and the fact that the Lie module map $\widehat{m_{2}}$ is a $\mathbf{B}$-derivation source.

If the graded module $V$ is a module over the graded commutative algebra $W$, then the tensor coalgebras $\mathrm{T}^{c} V$ and $\mathrm{T}^{c}(s V)$ are modules over $\mathrm{T} W$. We will follow our general convention and denote $w_{1} v_{1} \otimes \cdots \otimes w_{n} v_{n}$ by $w v_{[1 \text { to } n]}$ and, in the suspended setting, denote $w_{1} s v_{1} \otimes \cdots \otimes w_{n} s v_{n}$ by $w s v_{[1 \text { to } n]}$. The unraveling map

$$
\begin{aligned}
\widehat{\mathrm{U}}: \mathrm{T}^{c}(s V) & \rightarrow W \otimes \mathrm{T}^{c}(s V) \\
w s v_{[1 \text { to } n]} & \mapsto \widehat{\mathrm{u}}([1 \text { to } n]) w_{[1 \text { to } n]}^{\text {; }} \otimes s v_{[1 \text { to } n]},
\end{aligned}
$$

where $\widehat{\mathrm{u}}\left(\left[\begin{array}{ll}1 \text { to } & n\end{array}\right]\right)$ is the sign produced when moving the $w_{i}$ 's past the $v_{j}$ 's. The map $\widehat{\mathrm{U}}$ respects the coalgebra structure of $\mathrm{T}^{c}(s V)$.

Definition 2.4. A map $\widehat{f_{n}}:(s V)^{\wedge n} \rightarrow W$ is $W$-linear if

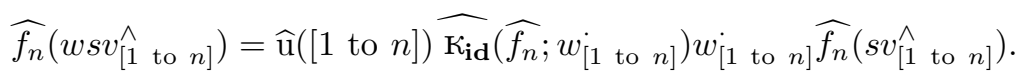

Likewise, a map $\widehat{m_{n}}:(s V)^{\wedge n-1} \otimes W \rightarrow W$ is $W$-linear if

$$
\begin{aligned}
& \widehat{m_{n}}\left(w s v_{[1 \text { to } n-1]}^{\wedge} \otimes w\right)= \\
& \widehat{\mathrm{u}}([1 \text { to } n-1]) \widehat{\mathrm{K}_{\mathbf{i d}}}\left(\widehat{m_{n}} ; w_{[1 \text { to } n-1]}^{\cdot}\right) w_{[1 \text { to } n-1]}^{\cdot} \widehat{m_{n}}\left(s v_{[1 \text { to } n-1]}^{\wedge} \otimes w\right) .
\end{aligned}
$$

For a map $\widehat{m_{n}}:(s V)^{\wedge n-1} \otimes W \rightarrow W$, we define the $s V$-extension map ${\widehat{m_{n}}}^{e}$ : $(s V)^{\wedge n} \rightarrow s V$ by setting

$$
{\widehat{m_{n}}}^{e}\left(w s v_{[1 \text { to } n]}^{\wedge}\right)=\sum_{\substack{\rho \\(n-1,1)}} \widehat{K_{\mathbf{i d}}}(\rho) \widehat{m_{n}}\left(w s v_{\rho[1 \text { to } n-1]} \otimes w_{\rho(n)}\right) s v_{\rho(n)} .
$$

Definition 2.5. Given $\widehat{m_{n}} \in \operatorname{Coder}^{W}(\bigwedge(s V) \otimes W)$, an $W$-linear, $W$-derivation source which is subordinate to $\widehat{l_{n}} \in \operatorname{Coder}^{W}(\bigwedge(s V))$, the map $\widehat{l_{n}}$ is said to rest on 


$$
\begin{aligned}
& \left.\widehat{m_{n}} \text { if } \widehat{l_{n}}\left(w s v_{[1}^{\wedge} \text { to } n\right]\right)= \\
& \quad \widehat{\mathrm{u}}([1 \text { to } n]) \widehat{\mathrm{K}_{\mathbf{i d}}}\left(\widehat{l_{n}} ; w_{[1 \text { to } n]}^{\cdot}\right) w_{[1 \text { to } n]} \widehat{l_{n}}\left(s v_{[1 \text { to } n]}^{\wedge}\right)+\widehat{m_{n}}\left(w s v_{[1 \text { to } n]}^{\wedge}\right) .
\end{aligned}
$$

The subset of $\operatorname{Coder}^{W}(\bigwedge(s V))$ of all coderivations $\widehat{l_{p}}$ which rest on $W$-linear $W$ derivation sources $\widehat{m_{p}}$ forms a graded Lie subalgebra. This subalgebra is denoted by $\operatorname{Coder}_{W}^{W}(\bigwedge(s V))$. Similarly, the subset of $\operatorname{Coder}^{W}(\bigwedge(s V) \otimes W)$ consisting of all $W$-linear $W$-derivation sources $\widehat{m_{p}}$ subordinate to maps $\widehat{l_{p}}$, which in turn rest on $\widehat{m_{p}}$, forms a graded Lie subalgebra, denoted by $\operatorname{Coder}_{W}^{W}(\bigwedge(s V) \otimes W)$.

Proposition 2.6. Let $\widehat{f_{n}}:(s V)^{\wedge n} \rightarrow W$ be a $W$-linear map and suppose the map $\widehat{m_{p}}:(s V)^{\wedge p-1} \otimes W \rightarrow W$ is a $W$-linear, $W$-derivation source subordinate to the map $\widehat{l_{p}}:(s V)^{\wedge p} \rightarrow V$, which in turn rests on $\widehat{m_{p}}$. Then $\left\langle\widehat{m_{p}}, \widehat{f_{n}}\right\rangle=\widehat{m_{p}} \widehat{f_{n}}-$ $\widehat{\kappa_{\text {id }}}\left(\widehat{m_{p}} ; \widehat{f_{n}}\right) \widehat{f_{n}} \widehat{l_{p}}$ is $W$-linear.

Proof of Proposition 2.6. In this proof and in the proofs to follow, we include only those sign arguments which are instructive.

First we compute $\widehat{m_{p}} \widehat{f_{n}}\left(w s v_{[1}\right.$ to $\left.\left.p+n-1\right]\right)$. We break up the result into two sums. The first one, $\left(\operatorname{Lin} \widehat{m_{p}} \widehat{f_{n}}\right)$, consists of those terms in which all the $w_{i}$ 's can and have been factored out. The remaining terms form the second sum (Nonlin $\widehat{m_{p}} \widehat{f_{n}}$ ). The first sum is over all $(p-1, n)$-unshuffles $\sigma$ :

$$
\left(\operatorname{Lin} \widehat{m_{p}} \widehat{f_{n}}\right)=\sum_{\sigma} \pm w_{[1 \text { to } p+n-1]} \widehat{m_{p}}\left(s v_{\sigma[1 \text { to } p-1]}^{\wedge} \otimes \widehat{f_{n}}\left(s v_{\sigma[p \text { to } n+p-1]}^{\wedge}\right)\right. \text {. }
$$

The second sum is actually a double sum over all $(p-1, n)$-unshuffles $\sigma$ and all $(1, n-1)$-unshuffles $\gamma$ acting on the second $\sigma$-hand:

$$
\begin{aligned}
& \text { (Nonlin } \left.\widehat{m_{p}} \widehat{f_{n}}\right)=\sum_{\sigma} \sum_{\gamma} \\
& \quad \pm \widehat{m_{p}}\left(s v_{\sigma[1 \text { to } p-1]}^{\wedge} \otimes w_{\sigma[1 \text { to } p-1]}^{\wedge} \cdot w_{\gamma \sigma[p \text { to } p+n-1]}^{\cdot}\right) \cdot \widehat{f_{n}}\left(s v_{\gamma \sigma[p \text { to } n+p-1]}^{\wedge}\right) .
\end{aligned}
$$

We compute $\widehat{f_{n}} \widehat{l_{p}}\left(w s v_{[1}^{\wedge}\right.$ to $\left.\left.p+n-1\right]\right)$ by again breaking it up into two sums: (Lin $\left.\widehat{f_{n}} \widehat{l_{p}}\right)$, is a sum over all $(p, n-1)$-unshuffles $\rho$, i.e.,

$$
\left(\operatorname{Lin} \widehat{f_{n} \hat{l}_{p}}\right)=\sum_{\rho} \pm w_{[1 \text { to } p+n-1]} \widehat{f_{n}}\left(\widehat{l_{p}}\left(s v_{\sigma[1 \text { to } p]}^{\wedge}\right) \wedge s v_{\sigma[p+1 \text { to } n+p-1]}^{\wedge}\right)
$$

and (Nonlin $\left.\widehat{f_{n}} \widehat{l_{p}}\right)$ is again a double sum over all $(p, n-1)$-unshuffles $\rho$ and all $(n-1,1)$-unshuffles $\tau$ acting on the first $\rho$-hand. In other words,

$$
\begin{aligned}
& \left(\text { Nonlin } \widehat{f_{n}} \widehat{l_{p}}\right)=\sum_{\rho} \sum_{\tau} \\
& \pm \widehat{m_{p}}\left(s v_{\tau \rho[1 \text { to } p-1]}^{\wedge} \otimes w_{\tau \rho[1 \text { to } p]}^{\cdot} \cdot w_{\rho[p+1 \text { to } p+n-1]}^{\cdot} \widehat{f_{n}}\left(s v_{\tau \rho(p)} \wedge s v_{\rho[p+1 \text { to } n+p-1]}^{\wedge}\right) .\right.
\end{aligned}
$$


Recognizing that $\left\|\widehat{m_{p}} \widehat{f_{n}}\right\|=\left\|\widehat{f_{n}} \widehat{l_{p}}\right\|$, it follows that

$$
\left[\left(\operatorname{Lin} \widehat{m_{p}} \widehat{f_{n}}\right)-\widehat{\kappa}\left(\widehat{m_{p}} ; \widehat{f_{n}}\right)\left(\operatorname{Lin} \widehat{f_{n}} \widehat{l_{p}}\right)\right]= \pm w_{[1 \text { to } p+n-1]}^{\cdot}\left\langle\widehat{m_{p}}, \widehat{f_{n}}\right\rangle\left(s v_{[1 \text { to } p+n-1]}^{\wedge}\right) \text {. }
$$

Therefore, once we show that $\left[\left(\right.\right.$ Nonlin $\left.\widehat{m_{p}} \widehat{f_{n}}\right)-\widehat{\kappa}\left(\widehat{m_{p}} ; \widehat{f_{n}}\right)\left(\right.$ Nonlin $\left.\left.\widehat{f_{n}} \widehat{l_{p}}\right)\right]=0$, the proof will be complete.

For every pair consisting of a $(p-1, n)$-unshuffle $\sigma$ and a $(1, n-1)$-unshuffle $\gamma$ acting on the second $\sigma$ hand, there is a unique pair consisting of a $(p, n-1)$-unshuffle $\rho$ and a $(p-1,1)$-unshuffle $\tau$ acting on the first $\rho$ hand which produces the exact same sequence of components, that is to say,

$$
\begin{aligned}
\sigma[1 \text { to } p-1] & =\tau \rho[1 \text { to } p-1], \\
\gamma \sigma(p) & =\tau \rho(p) \text { and } \\
\gamma \sigma[p+1 \text { to } p+n-1] & =\rho[p+1 \text { to } p+n-1] .
\end{aligned}
$$

Why should this be so? A simple counting argument provides the answer. Either combination produces an $(p-1,1, n-1)$-unshuffle. There are $\left(\begin{array}{c}p+n-2 \\ p-1\end{array}\right)$ distinct $(p-$ $1,1, n-1)$-unshuffles which send $j \in\{1, \ldots, p+n-1\}$ to the $p^{\text {th }}$ position because once the $p^{\text {th }}$ position is determined, the remaining $p+n-2$ terms must be unshuffled into a hand of length $p-1$ and a hand of length $n-1$. How many distinct pairs $(\sigma, \gamma)$ send $j$ to the $p^{\text {th }}$ position? This question is equivalent to asking how many distinct $(p-1, n)$-unshuffles $\sigma$ send $j$ to the second hand since, once $j$ is in the second hand, there is a unique $(1, n-1)$ unshuffle $\gamma$ which will send $j$ to the $p^{\text {th }}$ position. The number of $(p-1, n)$-unshuffles is $\left(\begin{array}{c}p+n-1 \\ n\end{array}\right)$ and $\left(\begin{array}{c}p+n-2 \\ n-1\end{array}\right)$ of them have $j$ in the second hand. Since $\left(\begin{array}{c}p+n-2 \\ n-1\end{array}\right)=\left(\begin{array}{c}p+n-2 \\ p-1\end{array}\right)$, we know there is a one-to-one correspondence between $(p-1,1, n-1)$-unshuffles and pair $(\sigma, \gamma)$ which send $j$ to the $p^{\text {th }}$ position. A similar argument shows that the number of $(p-1,1, n-1)$ unshuffles which send $j$ to the $p^{\text {th }}$ position equals the number of $(\rho, \tau)$ combinations which do the same. We can show that the $(\sigma, \gamma)$ term of (Nonlin $\left.\widehat{m_{p}} \widehat{f_{n}}\right)$ and the corresponding $(\rho, \tau)$ term of (Nonlin $\left.\widehat{f_{n}} \widehat{l_{p}}\right)$ have opposite signs, so they cancel.

Naturally, the graded commutative algebra $\operatorname{Hom}_{W}(\bigwedge(s V), \bigwedge(s V) \otimes W)$ is a shared Lie module over the Lie algebras $\operatorname{Coder}_{W}^{W}(\bigwedge(s V), \bigwedge(s V) \otimes W)$ and $\operatorname{Coder}_{W}^{W}(\bigwedge(s V))$.

\section{Lie algebras, their modules and cohomology in the coal- gebra setting}

\section{Lie algebras and their modules}

The most familiar definition of a Lie algebra $\mathfrak{g}$ is as an ungraded $k$-vector space (or, more generally, a module over a $k$-algebra $A$ ) equipped with a skew-symmetric bracket $l_{2}=[$,$] that maps \mathfrak{g} \otimes \mathfrak{g}$ into $\mathfrak{g}$ and that satisfies the Jacobi identity, i.e., the equality

$$
l_{2}\left(x, l_{2}(y, z)\right)=l_{2}\left(l_{2}(x, y), z\right)+l_{2}\left(y, l_{2}(x, z)\right)
$$


holds for all $x, y, z \in \mathfrak{g}$. In the context of graded coassociative coalgebras, we can consider $\mathfrak{g}$ a graded vector space where all elements of $\mathfrak{g}$ have combined degree $\langle 1,0\rangle$. The bracket $l_{2}$ becomes a degree $\langle-1,0\rangle$ coderivation on $\mathbb{Q} \mathfrak{g}$. No information is lost in the process because

$$
\begin{aligned}
l_{2}^{\otimes}(x \otimes y) & =l_{2}\left(x \otimes y+(-1)^{\langle 0,1\rangle \cdot\langle 0,1\rangle} y \otimes x\right) \\
& =l_{2}(x \otimes y)-l_{2}(y \otimes x) \\
& =l_{2}(x \otimes y)+l_{2}(x \otimes y) \\
& =2 l_{2}(x \otimes y) .
\end{aligned}
$$

But we can also define the Lie algebra on $\bigwedge(s \mathfrak{g})$, where the bracket $\widehat{l_{2}}$ becomes an internal graded symmetric coderivation of degree -1 on $\bigwedge(s \mathfrak{g})$ with respect to the internal grading. Again, no essential information is lost. We can therefore realize a Lie algebra in three equivalent ways:

1. A Lie algebra is a vector space $\mathfrak{g}$ over $k$ with skew symmetric bracket $l_{2}$ which satisfies the Jacobi identity (the original definition).

2. A Lie algebra is the subcoalgebra $\ \mathfrak{g}$, together with a combined degree $\langle-1,0\rangle$ coderivation $l_{2}$ satisfying $l_{2} \circ l_{2}=\frac{1}{2}\left[l_{2}, l_{2}\right]=0$, which is equivalent to the Jacobi identity. Since the internal degree is zero, it can be ignored, so $l_{2}$ can be considered a degree -1 coderivation, i.e., a differential on $\mathbb{Q g}$.

3. A Lie algebra is a subcoalgebra $\bigwedge(s \mathfrak{g})$ together with a suspended internal degree -1 differential $\widehat{l_{2}}$. This is our preferred setting.

The Jacobi identity is a result of the more general fact, true for all coderivations $\widehat{l}$ with odd degree, that $[\widehat{l}, \widehat{l}]=2 \widehat{l} \circ \widehat{l}$ and $[\widehat{l}, \widehat{l}]=-[\widehat{l}, \widehat{l}]=0$. In other words, any degree -1 coderivation $\hat{l}$ is a differential on the coalgebra $\Lambda(s \mathfrak{g})$ and if the differential $\widehat{l}$ has external degree -1 , then it is the extension of a map $\widehat{l}: s \mathfrak{g} \wedge s \mathfrak{g} \rightarrow s \mathfrak{g}$ as a coderivation, i.e., $\widehat{l}$ is a Lie bracket.

Suppose $\mathbf{B}$ is an ungraded Lie module over $\mathfrak{g}$, i.e., there is a map $\widehat{m_{2}}: s \mathfrak{g} \otimes \mathbf{B} \rightarrow \mathbf{B}$ such that $\widehat{m_{2}}\left(\widehat{l_{2}}(s x \wedge s y) \otimes b\right)-\widehat{m_{2}}\left(s x \otimes \widehat{m_{2}}(s y \otimes b)\right)+\widehat{m_{2}}\left(s y \otimes \widehat{m_{2}}(s x \otimes b)\right)=0$, for all $x, y \in \mathfrak{g}$ and $b \in \mathbf{B}$. The map $\widehat{m_{2}}$ is $\widehat{l_{2}}$-subordinate, and we can extend $\widehat{m_{2}}$ to an $\widehat{l_{2}}$-subordinate coderivation on $\Lambda(s \mathfrak{g}) \otimes \mathbf{B}$. The equation above can rewritten as $\widehat{m_{2}} \circ \widehat{m_{2}}=\frac{1}{2}\left[\widehat{m_{2}}, \widehat{m_{2}}\right]=0$ and since the degree of $\widehat{m_{2}}$ is -1 , it is a differential on $\bigwedge(s \mathfrak{g}) \otimes \mathbf{B}$. In the coalgebra setting, then, a $\mathfrak{g}$-module structure is any coderivation $\widehat{m_{2}}$ on $\Lambda(s \mathfrak{g}) \otimes \mathbf{B}$ which is a differential and which is subordinate to the bracket $\widehat{l_{2}}$.

\section{Chevalley-Eilenberg Cohomology}

Definition 3.1. A Chevalley-Eilenberg pair $(\mathbf{B}, \mathfrak{g})$ is a Lie algebra $\mathfrak{g}$ and Lie module B over $\mathfrak{g}$.

For any Chevalley-Eilenberg pair $(\mathbf{B}, \mathfrak{g})$, an $n$-multilinear function $f_{n}: \mathfrak{g}^{\times n} \rightarrow \mathbf{B}$ is alternating if

$$
f_{n}\left(x_{\sigma(1)}, \ldots, x_{\sigma(n)}\right)=(-1)^{\sigma} f_{n}\left(x_{1}, \ldots, x_{n}\right)
$$


for all $\sigma \in \Sigma_{n}$, where $(-1)^{\sigma}$ is the sign of the permutation. The algebra of all alternating multilinear functions $A_{l} t_{k}(\mathfrak{g}, \mathbf{B})$ is graded by $n$ and admits a degree +1 differential $\delta_{C E}: A l t_{k}^{n}(\mathfrak{g}, \mathbf{B}) \rightarrow A l t_{k}^{n+1}(\mathfrak{g}, \mathbf{B})$ given by equation (2). The cohomology of this complex with respect to $\delta_{C E}$ is the Chevalley-Eilenberg cohomology of $\mathfrak{g}$ with coefficients in $\mathbf{B}$.

In the coalgebra setting, any alternating $n$-multilinear function $f_{n}$ can be realized uniquely as a linear function $\widehat{f_{n}}:(s \mathfrak{g})^{\wedge n} \rightarrow \mathbf{B}$, which then can be extended as a coderivation $\widehat{f_{n}}: \bigwedge(s \mathfrak{g}) \rightarrow \bigwedge(s \mathfrak{g}) \otimes \mathbf{B}$. We extend $b \in \mathbf{B}$ as a coderivation

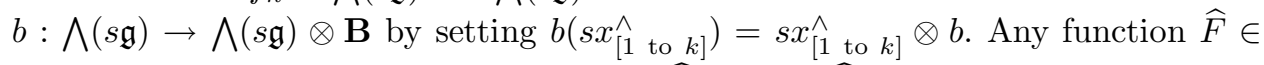
$\operatorname{Hom}(\bigwedge(s \mathfrak{g}), \bigwedge(s \mathfrak{g}) \otimes \mathbf{B})$ is the sum $\sum_{n=0}^{\infty} \widehat{f_{n}}$, where $\widehat{f_{n}}:(s \mathfrak{g})^{\wedge n} \rightarrow \mathbf{B}$ has degree $n$. Hence, $\operatorname{Hom}(\bigwedge(s \mathfrak{g}), \bigwedge(s \mathfrak{g}) \otimes \mathbf{B})$ is graded by $\mathbb{N}$ and is isomorphic to $\operatorname{Alt}_{k}(\mathfrak{g}, \mathbf{B})$. The differential $\delta_{C E}$ on $\operatorname{Hom}(\bigwedge(s \mathfrak{g}), \bigwedge(s \mathfrak{g}) \otimes \mathbf{B})$ has the elegant form $\left\langle\widehat{m_{2}},\right\rangle$. This formula works even for $b \in \mathbf{B}$, since $\delta_{C E} b=\left\langle\widehat{m_{2}}, b\right\rangle=\widehat{m_{2}} b+b \widehat{l_{2}}$ and $\widehat{l_{2}}=0$ on $s \mathfrak{g}$. Since $\left[\widehat{m_{2}}, \widehat{m_{2}}\right]=-\left[\widehat{m_{2}}, \widehat{m_{2}}\right]=0$, it is now simple to show that

$$
\delta_{C E} \circ \delta_{C E}=\left\langle\widehat{m_{2}}\left\langle\widehat{m_{2}}, \quad\right\rangle=\left\langle\frac{1}{2}\left[\widehat{m_{2}}, \widehat{m_{2}}\right],\right\rangle=0 .\right.
$$

If $\mathbf{B}$ is an algebra, then $\operatorname{Hom}(\bigwedge(s \mathfrak{g}), \bigwedge(s \mathfrak{g}) \otimes \mathbf{B})$ is also an algebra. The differential $\delta_{C E}$ acts as a derivation with respect to the multiplication.

\section{Lie-Rinehart pairs and Rinehart cohomology}

Suppose $\mathbf{B}$ is an algebra over $k$ which is also a $\mathfrak{g}$-module. The definition of a LieRinehart pair below has been modified from definition 1.1 to reflect the coalgebra setting.

Definition 3.2. [Rin63] Let $\mu: \mathbf{B} \otimes(s \mathfrak{g}) \rightarrow(s \mathfrak{g})$ be a left $\mathbf{B}$-module action on $s \mathfrak{g}$ denoted by $\mu(a \otimes \alpha):=a \alpha$. Let $\widehat{m_{2}}:(s \mathfrak{g}) \otimes \mathbf{B} \rightarrow \mathbf{B}$ be an $\widehat{l_{2}}$-subordinate $\mathbf{B}$-derivation source, i.e., an sa-module action on $\mathbf{B}$. The pair $(\mathbf{B}, s \mathfrak{g})$ is a Lie-Rinehart pair, provided the Lie-Rinehart relations (LRa) and (LRb) are satisfied.

LRa: $\widehat{m_{2}}$ is $\mathbf{B}$-linear.

LRb: $\widehat{l_{2}}$ rests on $\widehat{m_{2}}$ (definition 2.5).

Suppose $\widehat{f_{n}}$ is in $\operatorname{Hom}_{\mathbf{B}}(\bigwedge(s \mathfrak{g}), \bigwedge(s \mathfrak{g}) \otimes \mathbf{B})$. ¿From proposition 2.6, we know the image of $\widehat{f_{n}}$ under the Chevalley-Eilenberg differential $\delta_{C E}=\left\langle\widehat{m_{2}}, \quad\right\rangle$ is again $\mathbf{B}$ linear. The Rinehart algebra $R=\operatorname{Hom}_{\mathbf{B}}(\bigwedge(s \mathfrak{g}), \bigwedge(s \mathfrak{g}) \otimes \mathbf{B})$ with differential $\delta_{R}=$ $\left\langle\widehat{m_{2}}, \quad\right\rangle$ is a subcomplex of the Chevalley-Eilenberg complex and the cohomology of $R$ with respect to $\delta_{R}$ is the Rinehart cohomology of $\mathfrak{g}$ with coefficients in $\mathbf{B}$.

\section{Strongly homotopy Lie algebras, their modules and coho- mology}

Strongly homotopy Lie algebras (shLie algebras) first appeared implicitly in [Sul77] and explicitly in [SS] in the context of deformation theory. A concise introduction to shLie algebras is found in [LM95]. The initial definitions below are lifted directly from [LM95] and then modified to fit the language introduced in $\S 2$. 
Definition 4.1. [LM95] and [LS93] An $\mathcal{L}(m)$-structure on a graded module $L$ is a system of linear maps $\left\{l_{k}: 1 \leqslant k \leqslant m \leqslant \infty, k \neq \infty\right\}$, where $l_{k}: \otimes^{k} L \rightarrow L$ has combined degree $\langle 1-k, k-2\rangle$ and each map is combined graded symmetric in the sense that

$$
\left.\left.l_{k}\left(v_{\sigma[1} \text { to } k\right]\right)=\kappa_{\|\|}(\sigma) l_{k}\left(v_{[1} \text { to } k\right]\right)
$$

for all $\sigma \in \Sigma_{k}$. Moreover, the following generalized form of the Jacobi identity, the $n^{\text {th }}$ Jacobi identity map, is satisfied for $n \leqslant m$ :

$$
\left.\left.\mathcal{J}_{\mathrm{ID}_{n}}\left(v_{[1 \text { to } n]}\right)=\sum_{\substack{i+j=n+1 \\ i, j \geqslant 1}} \sum_{\sigma} \kappa_{\|\|}(\sigma)(-1)^{i(j-1)} l_{j}\left(l_{i}\left(v_{\sigma[1} \text { to } i\right]\right) \otimes v_{\sigma[i+1} \text { to } n\right]\right)=0,
$$

where the second summation runs over all $(i, j-1)$-unshuffles. The graded vector space $L$ is a strongly homotopy Lie algebra (or shLie algebra) if $L$ admits an $\mathcal{L}(\infty)$ structure.

We can rewrite this definition in terms of maps on $₫ L$ because the maps $l_{k}$ are combined graded symmetric. The Jacobi identity maps $\mathcal{J}_{\mathrm{ID} n}$ should equal 0 on $\Phi L$, but it is not immediately evident that the maps $\mathcal{J}_{\mathrm{ID} n}$ can be extended as coderivations on $\mathbb{Q} L$. Fortunately, we can rewrite the Jacobi identity maps using the bracket on $\operatorname{Coder}(\Phi L)$. Consider the bracket of $l_{j}$ with $l_{i}$ with respect to the combined grading on $₫ L$ :

$$
\left[l_{j}, l_{i}\right]=l_{j} l_{i}-(-1)^{\langle 1-j, j-2\rangle \cdot\langle 1-i, i-2\rangle} l_{i} l_{j}=l_{j} l_{i}+(-1)^{j+i} l_{i} l_{j} .
$$

Multiplying both sides by the desired sign for $l_{j} l_{i}$ in the Jacobi identity map $\mathcal{J}_{\operatorname{ID} n}$, we find that

$$
\begin{aligned}
(-1)^{i(j-1)}\left[l_{j}, l_{i}\right] & =(-1)^{i(j-1)} l_{j} l_{i}+(-1)^{i(j-1)+j+i} l_{i} l_{j} \\
& =(-1)^{i(j-1)} l_{j} l_{i}+(-1)^{j(i-1)} l_{i} l_{j} .
\end{aligned}
$$

We therefore reformulate the definition of an $\mathcal{L}(m)$-algebra and an shLie algebra:

Definition 4.2. An $\mathcal{L}(m)$-structure on a graded module $L$ consists of a system of coderivations $\left\{l_{k}: \mathbb{Q} L \rightarrow \mathbb{Q} L: 1 \leqslant k \leqslant m \leqslant \infty, k \neq \infty\right\}$, where each $l_{k}$ has combined degree $\langle 1-k, k-2\rangle$. Moreover, the following generalized form of the Jacobi identity is satisfied for $n \leqslant m$ :

$$
\mathcal{J}_{\mathrm{ID} n}=\frac{1}{2} \sum_{\substack{i+j=n+1 \\ i, j \geqslant 1}}(-1)^{i(j-1)}\left[l_{j}, l_{i}\right]=0
$$

on $L \otimes n$. Again, if $L$ admits an $\mathcal{L}(\infty)$-structure, then $L$ is a strongly homotopy Lie algebra (an shLie algebra).

Since the Jacobi identity maps are sums of coderivations, it follows that $\mathcal{J}_{\mathrm{ID} n}$ can be extended to a coderivation on $\mathbb{Q} L$. Furthermore, since $\mathcal{J}_{\mathrm{ID} n}=0$ on $L \otimes n$, we have proven the following proposition. 
Proposition 4.3. $\mathcal{J}_{\mathrm{ID}_{n}}=0$ on $\ L$.

In both [LS93] and [LM95], this result was not achieved without first passing to the suspended internal graded symmetric setting. Lada, Markl and Stasheff defined strongly homotopy Lie algebras on the tensor coalgebra $T^{c} L$ rather than on $\mathbb{Q} L$, whose existence had not been recognized. The maps $\mathcal{J}_{\mathrm{ID} n}$ cannot be extended as coderivations on the tensor coalgebra while preserving the desired symmetry. So instead, they suspended the maps $l_{k}$, changing the symmetry of the maps from combined graded symmetric to suspended internal graded symmetric. They were then in familiar territory, where the maps $\widehat{\mathcal{J}_{\mathrm{ID} n}}$ could be extended as coderivations on $\bigwedge(s L)$, even though they did not make explicit use of the bracket on $\operatorname{Coder}(\bigwedge(s L))$.

\section{Modules over a strongly homotopy Lie algebra}

Definition 4.4. [LM95] Let $L=\left(L, l_{i}\right)$ be an $\mathcal{L}(p)$-algebra $(0<p<\infty)$ and let $M$ be a differential graded module with differential $m_{1}$. Then a left $\mathcal{L}(k)$-module structure over $L$ on $M$ (for $k \leqslant p$ ) is a collection $\left\{m_{n}: 1 \leqslant n \leqslant k, n \neq \infty\right.$ of $l_{n}$-subordinate coderivations $m_{n}$ on $\ L \otimes M$ such that the $n^{\text {th }}$ action identity map

$$
\begin{aligned}
\mathcal{A} C T_{\mathrm{ID} n} & =\sum_{i+j=n+1}(-1)^{i(j-1)} m_{j} m_{i} \\
& =\frac{1}{2} \sum_{\substack{i+j=n+1 \\
i, j \geqslant 1}}(-1)^{i(j-1)}\left[m_{j}, m_{i}\right] \\
& =0
\end{aligned}
$$

on $\ L \otimes M$. The differential graded module $M$ is a strongly homotopy Lie module over $L$ (or an $L$-shLie module) if $L$ admits an $\mathcal{L}(\infty)$-structure and $M$ is a module with respect to that $\mathcal{L}(\infty)$-structure.

Definition 4.4 implies that the differential $m_{1}$ on $M$ must be $l_{1}$-subordinate. It is simple to verify that $m_{1}$ is a differential on $\mathbb{Q} L \otimes M$.

When $M$ is a differential graded commutative algebra, we require that the maps $m_{i}$ be $M$-derivation sources, that is to say, for every $v_{[1 \text { to } i-1]}^{\mathbb{Q}} \in L^{\otimes i-1}$, the map $m_{i}\left(v_{[1}\right.$ to $\left.\left.i-1\right] \otimes(\quad)\right): M \rightarrow M$ is in $\operatorname{Der}(M)$.

Since $\operatorname{Coder}(\triangle L) \approx \operatorname{Coder}(\bigwedge(s L))$, it follows that if $\left(L, l_{i}\right)$ is a strongly homotopy Lie algebra, then $\left(s L, \widehat{l_{i}}\right)$, where $\widehat{l_{i}}$ is the coderivation on $\bigwedge(s L)$ induced by the isomorphism $S: \mathbb{Q} L \rightarrow \bigwedge(s L)$, is also an shLie algebra. The suspended internal degree of each map $\widehat{l_{i}}$ is -1 , so the sum of the $\mathcal{L}(\infty)$-structure maps $\widehat{l_{i}}$ is a differential on $\bigwedge(s L)$, first shown in [LS93]. This is not the case for $\left(L, l_{i}\right)$ because each $l_{i}$ is bigraded. In order for the sum of the $l_{i}$ 's to be a differential, one would have to make sense of $l_{i}$ as a degree -1 map. Passing to the suspended internal graded symmetric setting is a convenient way to accomplish this because degree of $\widehat{l_{i}}$ is $\mathbf{e d}\left(l_{i}\right)+\mathbf{i d}\left(l_{i}\right)=-1$, which is the total degree of $l_{i}$. For $\left(s L, \widehat{l_{i}}\right)$, the $n^{\text {th }}$ Jacobi 
identity map is

$$
\widehat{\mathcal{J}_{\mathrm{ID}} n}=\frac{1}{2} \sum_{\substack{i+j=n+1 \\ i, j \geqslant 1}}\left[\widehat{l_{j}}, \widehat{l_{i}}\right]
$$

which is 0 on $\bigwedge(s L)$.

Proposition 4.5. [LS93] If $\left(s L, \widehat{l_{i}}\right)$ is an shLie algebra, the map $\mathrm{D}_{s L}=\sum_{i=1}^{\infty} \widehat{l_{i}}$ is a differential on $\bigwedge(s L)$, i.e. it is a map of degree -1 such that $\mathrm{D}_{s L} \circ \mathrm{D}_{s L}=0$.

Proof of Proposition 4.5. A proof can be found in [LS93]. Here the proof is essentially the same but takes advantage of the bracket of coderivations:

$$
\mathrm{D}_{s L} \circ \mathrm{D}_{s L}=\left(\sum_{j=1}^{\infty} \widehat{l_{j}}\right) \circ\left(\sum_{i=1}^{\infty} \widehat{l_{i}}\right)=\sum_{j=1}^{\infty} \sum_{i=1}^{\infty} \widehat{l_{j}} \widehat{l_{i}}=\sum_{n=1}^{\infty} \sum_{\substack{i+j=n+1 \\ i, j \geqslant 1}} \widehat{l_{j}} \widehat{l_{i}} .
$$

Using equation (9) in definition 4.2, we see that

$$
\sum_{n=1}^{\infty} \sum_{\substack{i+j=n+1 \\ i, j \geqslant 1}} \widehat{l_{j}} \widehat{l_{i}}=\frac{1}{2} \sum_{n=1}^{\infty} \sum_{\substack{i+j=n+1 \\ i, j \geqslant 1}}\left[\widehat{l_{j}}, \widehat{l_{i}}\right]=\frac{1}{2} \sum_{n=1}^{\infty} \widehat{\mathcal{J}_{\mathrm{ID}}}=0 .
$$

Note also that $\mathrm{D}_{s L}$ is a degree -1 coderivation, so $\mathrm{D}_{s L} \circ \mathrm{D}_{s L}=\frac{1}{2}\left[\mathrm{D}_{s L}, \mathrm{D}_{s L}\right]$ and the graded skew-commutativity of the bracket ensures that $\left[\mathrm{D}_{s L}, \mathrm{D}_{s L}\right]=0$.

Suppose $\left(M, m_{i}\right)$ is an shLie module over $\left(L, l_{i}\right)$. In the suspended internal graded symmetric setting, the maps $\widehat{m_{i}}: \bigwedge(s L) \otimes M \rightarrow \bigwedge(s L) \otimes M$ form an shLie module structure on $M$ over $\left(s L, \widehat{l_{i}}\right)$. Again, the degree of $\widehat{m_{i}}$ is -1 , the total degree of $m_{i}$, and the action identity map has the form

$$
\widehat{\mathcal{A C T}} \widehat{\mathrm{ID}}_{n}=\frac{1}{2} \sum_{\substack{i+j=n+1 \\ i, j \geqslant 1}}\left[\widehat{m_{j}}, \widehat{m_{i}}\right]
$$

which is zero on $\bigwedge(s L) \otimes M$.

Proposition 4.6. The map $\mathrm{D}_{M}=\sum_{i=1}^{\infty} \widehat{m_{i}}$ is a differential on $\bigwedge(s L) \otimes M$, i.e., $\mathrm{D}_{M} \circ \mathrm{D}_{M}=0$.

Proof of Proposition 4.6. Isomorphic to the proof of proposition 4.5. Notice that $\mathrm{D}_{M}$ is $\mathrm{D}_{s L}$-subordinate and that $\mathrm{D}_{M} \circ \mathrm{D}_{M}=\frac{1}{2}\left[\mathrm{D}_{M}, \mathrm{D}_{M}\right]=0$.

It is only for historic reasons that we present shLie structures and shLie module structures first in the combined graded symmetric setting first and then in the suspended internal graded symmetric one. We will now use the suspended internal graded symmetric setting exclusively.

\section{Homotopy Chevalley-Eilenberg cohomology}

Definition 4.7. A homotopy Chevalley-Eilenberg pair $(M, L)$ consists of an shLie algebra $L$ and an L-shLie module $M$. 
A multi-linear alternating function $F_{n}: L^{\times n} \rightarrow M$ can be seen as a linear function $\widehat{F_{n}}:(s L)^{\wedge n} \rightarrow M$. So the homotopy Chevalley-Eilenberg algebra $\operatorname{Alt}_{k}(L, M)$ is isomorphic to

$$
\operatorname{Hom}_{k}(\bigwedge(s L), \bigwedge(s L) \otimes M) .
$$

Any homogeneous map $\widehat{F}$ splits into a sum $\sum_{n=0}^{\infty} \widehat{F}_{n}$, where $\widehat{F}_{n}:(s L)^{\wedge n} \rightarrow M$. The map $\widehat{F}_{n}$ is extended as a coderivation on $\bigwedge(s L) \otimes M$. Any function $\widehat{F}_{0}$ is an element $a \in M$. The degree of $a:(s L)^{\wedge 0} \rightarrow M$ as a map is the negative of its degree as an element of $M$. The map $a$ is extended as a coderivation on $\bigwedge(s L) \otimes M$ by setting

$$
a\left(s v_{[1 \text { to } k]}^{\wedge}\right)=\widehat{K_{\text {id }}}\left(a ; s v_{[1 \text { to } k]}^{\wedge}\right) s v_{[1 \text { to } k]}^{\wedge} \otimes a .
$$

Instead of $\mathbb{N}$-graded, as was the case in the ungraded setting, the Chevalley-Eilenberg complex $\operatorname{Hom}_{k}(\bigwedge(s L), \bigwedge(s L) \otimes M)$ is $\mathbb{Z}$-graded by suspended internal degree. For any $\widehat{F} \in \operatorname{Hom}_{k}(\bigwedge(s L), \bigwedge(s L) \otimes M)$ of degree $\widehat{\mathbf{i d}}(\widehat{F})$, we define

$$
\delta_{h C E} \widehat{F}=\mathrm{D}_{M} \widehat{F}-\widehat{K}_{\mathbf{i d}}(\widehat{F}) \widehat{F} \mathrm{D}_{s L}=\left\langle\mathrm{D}_{M}, \widehat{F}\right\rangle,
$$

which has degree $\widehat{\mathbf{i d}}(\widehat{F})+1$.

Proposition 4.8. $\delta_{h C E} \circ \delta_{h C E}=0$.

Proof of Proposition 4.8.

$$
\begin{aligned}
\delta_{h C E} \circ \delta_{h C E} & =\left\langle\mathrm{D}_{M},\left\langle\mathrm{D}_{M},\right\rangle\right\rangle \\
& =\left\langle\frac{1}{2}\left[\mathrm{D}_{M}, \mathrm{D}_{M}\right],\right\rangle \\
& =0 .
\end{aligned}
$$

The cohomology with respect to $\delta_{h C E}$ is the homotopy Chevalley-Eilenberg cohomology of $s L$ with coefficients in $M$.

When $M$ is a differential graded commutative algebra, the homotopy ChevalleyEilenberg complex $\operatorname{Hom}_{k}(\bigwedge(s L), \bigwedge(s L) \otimes M)$ is a differential graded commutative algebra. Suppose $\widehat{F}=\sum_{i=0}^{\infty} \widehat{F}_{i}$ and $\widehat{G}=\sum_{j=0}^{\infty} \widehat{G_{j}}$ are both elements of the complex, where $\widehat{F}_{i}: \bigwedge^{i}(s L) \rightarrow M$ and $\widehat{G_{j}}: \bigwedge^{j}(s L) \rightarrow M$. The product $\widehat{F} \smile \widehat{G}=\sum_{n=0}^{\infty}(\widehat{F} \smile$ $\widehat{G})_{n}$ is given by $(\widehat{F} \smile \widehat{G})_{n}=\sum_{i+j=n} \widehat{F}_{i} \smile \widehat{G_{j}}$. The differential $\delta_{h C E}$ acts as a derivation with respect to this multiplication.

\section{Homotopy Lie-Rinehart pairs and homotopy Rinehart cohomology}

The homotopy Rinehart complex is a straightforward generalization of the Rinehart complex in the ungraded setting. Since the Rinehart complex is defined only for LieRinehart pairs $(\mathbf{B}, \mathfrak{g})$, we must define what constitutes a homotopy Lie-Rinehart pair $(M, s L)$. The subset $A l t_{M}(s L, M)$ of $\operatorname{Alt}_{k}(s L, M)$ consisting of all $M$-linear alternating functions is isomorphic to $\operatorname{Hom}_{M}(\bigwedge(s L), \bigwedge(s L) \otimes M)$.

Definition 4.9. A homotopy Lie-Rinehart pair $(M, s L)$ consists of a differential graded commutative algebra $\left(M, \widehat{m_{i}}\right)$ which is an shLie module over the shLie algebra 
$\left(s L, \widehat{l_{i}}\right)$, which in turn is an $M$-module. Moreover, the following two homotopy LieRinehart relations must be satisfied for all $i \geqslant 1$ :

$\left(h L R a_{i}\right)$ : The shLie module structure map $\widehat{m_{i}}$ is $M$-linear.

$\left(h L R b_{i}\right)$ : The shLie structure map $\widehat{l_{i}}$ rests on $\widehat{m_{i}}$ (definition 2.5).

An $\mathcal{L}(p)$-Lie-Rinehart pair $\left(\left(M, \widehat{m_{i}}\right),\left(s L, \widehat{l_{i}}\right)\right)$ has maps $\widehat{m_{i}}$ and $\widehat{l_{i}}$ which satisfy $\left(h L R a_{i}\right)$ and $\left(h L R b_{i}\right)$ for $1 \leqslant i \leqslant p$.

Proposition 4.10. If $\widehat{F}$ is $M$-linear, then so is $\delta_{h C E} \widehat{F}$.

Proof of Proposition 4.10. The image of $\widehat{F}$ under $\delta_{h C E}$ is

$$
\left\langle\mathrm{D}_{M}, \widehat{F}\right\rangle=\sum_{i=1}^{\infty}\left\langle\widehat{m_{i}}, \widehat{F}\right\rangle \text {. }
$$

The proof follows as a consequence of proposition 2.6.

We conclude that the subset of all $M$-linear functions in $\operatorname{Hom}_{k}(\bigwedge(s L), \bigwedge(s L) \otimes$ $M)$ forms a subcomplex $\mathcal{R}=\operatorname{Hom}_{M}(\bigwedge(s L), \bigwedge(s L) \otimes M)$ with differential $\delta_{\mathcal{R}}=$ $\left\langle\mathrm{D}_{M},\right\rangle$. The differential, together with the cup product, provides the homotopy Rinehart complex $\mathcal{R}$ with the structure of a differential graded commutative algebra. The cohomology of $\mathcal{R}$ with respect to $\delta_{\mathcal{R}}$ is the homotopy Rinehart cohomology of $s L$ with coefficients in $M$.

\section{Homotopy Lie-Rinehart resolutions of Lie-Rinehart pairs}

It is often necessary to replace the components of a complex with resolutions of those component in order to construct a model for a complex with the same basic algebraic structure and precisely the same (co)homology as the original. (It is not always necessary to replace all of the components, see [Sta92], for example.) When building a model for the Rinehart cohomology for a Lie-Rinehart pair $(\mathbf{B}, s \mathfrak{g})$ over a $k$-algebra $A$, we replace the pair with a pair of resolutions $(M, s L)$, which retain as much of the algebraic structure of $(\mathbf{B}, s \mathfrak{g})$ as possible. It makes sense, then, that $(M, L)$ should form a homotopy Lie-Rinehart pair.

\section{Piecing together Lie-Rinehart resolutions}

Let $(\mathbf{B}, s \mathfrak{g})$ be a Lie-Rinehart pair over a $k$-algebra $A$ with module structure maps $\widehat{\mu}: \mathbf{B} \otimes s \mathfrak{g} \rightarrow s \mathfrak{g}$ and $\widehat{m_{2}}: s \mathfrak{g} \otimes \mathbf{B} \rightarrow \mathbf{B}$. Let $\widehat{l_{2}}$ denote the bracket on $s \mathfrak{g}$ and $\pi_{\mathbf{B}}$ denote the multiplication on $\mathbf{B}$. (Henceforth, only maps associated with the Lie-Rinehart pair will have hats.) The basic ingredients of a homotopy Lie-Rinehart resolution for a Lie-Rinehart pair $(\mathbf{B}, s \mathfrak{g})$ are $\left(s L, l_{i}\right)$ and $\left(M, m_{i}, \pi_{M}\right)$ where $\mathrm{H}_{l_{1}}(s L)=s \mathfrak{g}$ and $\mathrm{H}_{m_{1}}(M)=\mathbf{B}$.

Definition 5.1. A homotopy Lie-Rinehart resolution of a Lie-Rinehart pair $(\mathbf{B}, s \mathfrak{g})$ over an algebra $A$ is a homotopy Lie-Rinehart pair $(M, s L)$ over $A$, such that

(a) the shLie algebra $\left(s L, l_{i}\right)$, seen as the coalgebra $\Lambda(s L)$, resolves $\Lambda(s \mathfrak{g})$, i.e.,

$$
\mathrm{H}_{l_{1}}(\bigwedge(s L))=\bigwedge(s \mathfrak{g}),
$$


where (following the physicists' notation) $\mathrm{H}_{l_{1}}$ denotes the homology with respect to the differential $l_{1}$,

(b) the differential graded commutative algebra $\left(M, m_{i}, \pi_{M}\right)$ satisfies

$$
\mathrm{H}_{m_{1}}(\bigwedge(s L) \otimes M)=\bigwedge(s \mathfrak{g}) \otimes \mathbf{B},
$$

(c) the dgca $\left(M, m_{i}, \pi_{M}\right)$ also satisfies

$$
\mathrm{H}_{m_{1}}(M \otimes M)=\mathbf{B} \otimes \mathbf{B} .
$$

Furthermore, the following conditions hold:

i. $\mathrm{H}_{l_{1}}\left(l_{2}\right)=\widehat{l_{2}}$ on $\bigwedge(s \mathfrak{g})$.

ii. $\mathrm{H}_{m_{1}}\left(m_{2}\right)=\widehat{m_{2}}$ on $\bigwedge(s \mathfrak{g}) \otimes \mathbf{B}$.

iii. $\mathrm{H}_{m_{1}}\left(\pi_{M}\right)=\pi_{\mathbf{B}}$ on $\mathbf{B} \otimes \mathbf{B}$.

The conditions above are quite reasonable, but satisfying them is far from automatic. Consider any homotopy Lie-Rinehart pair $(M, s L)$. Since $m_{1}$ is a derivation on $M$, the multiplication on $M$ is a chain map which induces a well-defined map $\mathrm{H}_{m_{1}}\left(\pi_{M}\right)$ from $\mathrm{H}_{m_{1}}(M \otimes M)$ into $\mathrm{H}_{m_{1}}(M)$. Both $\bigwedge(s L)$ and $\bigwedge(s L) \otimes M$ are complexes with differentials $l_{1}$ and $m_{1}$, respectively. Since the maps $\mathcal{J}_{\mathrm{ID} 2}$ and $\mathcal{A} C T_{\mathrm{ID} 2}$ are both zero, it follows that both $l_{2}$ and $m_{2}$ are graded morphisms of complexes and as such, induce well-defined maps $\mathrm{H}_{l_{1}}\left(l_{2}\right)$ and $\mathrm{H}_{m_{1}}\left(m_{2}\right)$ on their respective homologies.

In the proposition below, we state conditions on the homotopy Lie-Rinehart pair which guarantees it satisfies the conditions in definition 5.1.

Proposition 5.2. Let $(M, s L)$ be a homotopy Lie-Rinehart pair. Suppose the differential graded algebra $\left(M, m_{i}, \pi_{M}\right)$ is a projective resolution of $\mathbf{B}$ over $A$ which respects the algebra structure on $\mathbf{B}$, i.e., condition iii of definition 5.1 is satisfied. Suppose $\left(s L, l_{i}\right)$ is a projective resolution sg. Furthermore,

(a) $\mathrm{H}_{l_{1}}\left(l_{2}\right)=\widehat{l_{2}}$ on $s \mathfrak{g} \wedge s \mathfrak{g}$ and

(b) $\mathrm{H}_{m_{1}}\left(m_{2}\right)=\widehat{m_{2}}$ on $s \mathfrak{g} \otimes \mathbf{B}$.

Then $(M, s L)$ is a homotopy Lie-Rinehart resolution of $(\mathbf{B}, s \mathfrak{g})$.

\section{SDR-Data}

For the proof of proposition 5.2, we will use strong deformation retract data (SDRdata). In general, $S D R$-data for two differential graded modules $\left(M, d_{M}\right)$ and $\left(N, d_{N}\right)$ over a commutative ring $R$ with unity consist of two degree 0 chain maps $\lambda: M \rightarrow N$ and $p: N \rightarrow M$ and a homotopy $h: N \rightarrow N$ such that

$$
\begin{aligned}
& p \lambda=1_{M} \quad \text { and } \\
& \lambda p=1_{N}+d_{N} h+h d_{N},
\end{aligned}
$$

which guarantee an isomorphism of homology (or cohomology), that is to say, $H_{d_{M}}(M) \approx H_{d_{N}}(N)$. SDR-data is succinctly described by

$$
\left(\left(M, d_{M}\right) \underset{p}{\stackrel{\lambda}{\rightleftarrows}}\left(N, d_{N}\right), h\right) \text {. }
$$


When the inclusion map $\lambda$ and the projection map $p$ also respect additional algebraic structure on $M$ and $N$, then that structure is carried over to homology (or cohomology).

Proof of Proposition 5.2. For the deleted projective resolution $M$ of $\mathbf{B}$, the nondeleted projective resolution $M^{+}: M \stackrel{p_{M}}{\rightarrow} \mathbf{B} \rightarrow 0$ is acyclic, so there exists a contracting homotopy $s_{i}:\left(M^{+}\right)_{i} \rightarrow\left(M^{+}\right)_{i+1}$ where $s_{-1}: \mathbf{B} \rightarrow M_{0}$ such that $1_{M^{+}}=m_{1}^{+} s+s m_{1}^{+}$[Wei94]. Setting $\lambda_{M}=s_{-1}$ and $-\left(h_{M}\right)_{i}=s_{i}$ for $i \geqslant 0$, we produce SDR-data

$$
\left((\mathbf{B}, 0) \underset{p_{M}}{\stackrel{\lambda_{M}}{\rightleftharpoons}}\left(M, m_{1}\right), h_{M}\right) .
$$

By construction, $p_{M} \lambda_{M}=1_{\mathrm{B}}$. On $M_{i}$ for $i>0$, the map $\lambda_{M} p_{M}=0$ and $1_{M_{i}}=$ $-m_{1} h_{M}-h_{M} m_{1}$. For $i=0$, the identity map $1_{M_{0}}=-h_{M} m_{1}+\lambda_{M} p_{M}$ and $m_{1} h_{M}=$ 0 . Therefore $\lambda_{M} p_{M}=1_{M}+m_{1} h_{M}+h_{M} m_{1}$. Likewise, we can construct SDR-data

$$
\left((s \mathfrak{g}, 0) \underset{p_{s L}}{\stackrel{\lambda_{s L}}{\rightleftarrows}}\left(s L, l_{1}\right), h_{s L}\right)
$$

for $s \mathfrak{g}$ and $s L$.

We will now repeatedly "tensor" SDR-data to produce new SDR-data, using the Gugenheim tensor trick ([GLS91]) for defining the homotopy $h$. We illustrate this technique by showing that

$$
\left((\mathbf{B} \otimes \mathbf{B}, 0) \underset{p_{M} \otimes p_{M}}{\stackrel{\lambda_{M} \otimes \lambda_{M}}{\rightleftarrows}}\left(M \otimes M, m_{1}\right), \quad h_{M \otimes M}\right),
$$

is SDR-data, where setting $h_{M \otimes M}=1_{M} \otimes h_{M}+h_{M} \otimes \lambda_{M} p_{M}$ was first proposed by Gugenheim and Lambe in [GL89]. Clearly, $\left(p_{M} \otimes p_{M}\right)\left(\lambda_{M} \otimes \lambda_{M}\right)=1_{\mathbf{B} \otimes \mathbf{B}}$ and it is a simple exercise to show that $1_{M \otimes M}+m_{1} h_{M \otimes M}+h_{M \otimes M} m_{1}=\left(\lambda_{M} \otimes \lambda_{M}\right)\left(p_{M} \otimes p_{M}\right)$. We have verified that equation (13) and condition (iii) in definition 5.1 hold.

The tensor trick can be extended to show that

$$
\left(\left(\mathrm{T}^{c}(s \mathfrak{g}), 0\right) \underset{\mathrm{T} p_{s L}}{\stackrel{\mathrm{T} \lambda_{s L}}{\rightleftarrows}}\left(\mathrm{T}^{c}(s L), l_{1}\right), \mathrm{T} h_{s l}\right)
$$

is SDR-data, where $\mathrm{T} \lambda_{s L}=\lambda_{s L}{ }^{\otimes i}$ on $(s \mathfrak{g})^{\otimes i}$, where $\mathrm{T} p_{s L}=p_{M}^{\otimes i}$ on $(s L)^{\otimes i}$ and where $\mathrm{T} h_{s L}=\sum_{i=1}^{\infty} \mathrm{T}_{i} h_{s L}$ with $\mathrm{T}_{i} h_{s L}=\sum_{j=1}^{i} 1^{\otimes j-1} \otimes h_{s L} \otimes\left(\lambda_{s L} p_{s L}\right)^{\otimes i-j}$ on $(s L)^{\otimes i}$ (see [GLS91]). It is clear that $\mathrm{T} p_{s L} \mathrm{~T} \lambda_{s L}=1_{\mathrm{T}^{c}(s \mathfrak{g})}$. We can show that $\mathrm{T} \lambda_{s L} \mathrm{~T} p_{s L}=1_{\mathrm{T}^{c}(s L)}+l_{1} \mathrm{~T} h_{s L}+\mathrm{T} h_{s L} l_{1}$ by induction on $n$. As a result, $\mathrm{H}_{l_{1}}\left(\mathrm{~T}^{c}(s L)\right)=$ $\mathrm{T}^{c}(s \mathfrak{g})$. Similarly, we can define SDR-data

$$
\left(\left(\mathrm{T}^{c}(s \mathfrak{g}) \otimes \mathbf{B}, 0\right) \underset{\mathrm{T} p_{M}}{\stackrel{\mathrm{T} \lambda_{M}}{\rightleftarrows}}\left(\mathrm{T}^{c}(s L) \otimes M, m_{1}\right), \mathrm{T} h_{M}\right)
$$


where $\mathrm{T} h_{M}=\sum_{i=1}^{\infty} \mathrm{T}_{i} h_{M}$ with

$$
\begin{aligned}
\mathrm{T}_{i} h_{M} & =\left(\sum_{j=1}^{i-1} 1^{\otimes j-1} \otimes h_{s L} \otimes\left(\lambda_{s L} p_{s L}\right)^{\otimes i-j+1} \otimes \lambda_{M} p_{M}\right)+1^{\otimes i-1} \otimes h_{M} \\
& =\mathrm{T}_{i-1} h_{s L} \otimes \lambda_{M} p_{M}+1^{\otimes i-1} \otimes h_{M}
\end{aligned}
$$

on $(s L)^{\otimes i-1} \otimes M$.

Besides the inclusion map $i: \bigwedge(s L) \rightarrow \mathrm{T}^{c}(s L)$, there is also a splitting map $j: \mathrm{T}^{c}(s L) \rightarrow \bigwedge(s L)$ which sends $s v_{[1 \text { to } n]}$ to $\frac{1}{n !} s v_{[1 \text { to } n]}^{\wedge}$. Both $i$ and $j$ are chain maps, so the composition $j i=1 \wedge(s L)$ induces an isomorphism between $\mathrm{H}_{l_{1}}(\bigwedge(s L))$ and $\bigwedge\left(\mathrm{H}_{l_{1}}(s L)\right)$, which equals $\bigwedge(s \mathfrak{g})$. Similarly, using $\left(i \otimes 1_{M}\right)$ and $\left(j \otimes 1_{M}\right)$, we can show that $\mathrm{H}_{m_{1}}(\bigwedge(s L) \otimes M)=\bigwedge(s \mathfrak{g}) \otimes \mathbf{B}$. Having verified that equations (11) and (12) in definition 5.1 hold, it is now straightforward to show that conditions (a) and (b) in proposition 5.2 guarantee that conditions (i) and (ii) are satisfied.

We can use a spectral sequence argument to prove the following result.

THEOREM 5.3. If the pair $(M, s L)$ is a projective homotopy Lie-Rinehart resolution of the Lie-Rinehart pair $(\mathbf{B}, s \mathfrak{g})$, then $\left(\mathcal{R},\left\langle\mathrm{D}_{M}, \quad\right\rangle\right)$ is a cohomological model for $\left(R,\left\langle\widehat{m_{2}},\right\rangle\right)$.

Proof of Proposition 5.3. We have the following SDR-data:

and

$$
\left((\bigwedge(s \mathfrak{g}), 0) \underset{p_{1}}{\stackrel{\lambda_{1}}{\rightleftharpoons}}\left(\bigwedge(s L), l_{1}\right), h_{1}\right)
$$

$$
\left((\bigwedge(s \mathfrak{g}) \otimes \mathbf{B}, 0) \underset{p_{2}}{\stackrel{\lambda_{2}}{\rightleftarrows}}\left(\bigwedge(s L) \otimes M, m_{1}\right), h_{2}\right) .
$$

The $E_{0}$ term of the spectral sequence is the algebra $\mathcal{R}$, bigraded by the external degree of a map and the difference between the internal degree and the external degree, e.g, if the map $F_{n}$ has internal degree $\left|F_{n}\right|$, then it has bidegree $\left(n,\left|F_{n}\right|-n\right)$. The differential $\left\langle\mathrm{D}_{M},\right\rangle$ splits up into the sum $\sum_{i=1}^{\infty}\left\langle m_{i},\right\rangle$, each of which has bidegree $(i-1,2-i)$. The map $\left\langle m_{1},\right\rangle$ is the differential on $E_{0}$. If $F_{n}$ is a cocycle, then $F_{n}$ is a chain map between the complexes $\bigwedge(s L)$ and $\bigwedge(s L) \otimes M$. So the class of $F_{n}$ induces a map $\left[F_{n}\right]: \bigwedge(s \mathfrak{g}) \rightarrow \bigwedge(s \mathfrak{g}) \otimes \mathbf{B}$. Therefore, the $E_{1}$ term is contained in $R$ and all elements $\left[F_{n}\right]$ of $E_{1}$, have bidegree $(n, 0)$. It follows that the cohomology of $\mathcal{R}$ is the cohomology of the $E_{1}$ term with respect to the differential $\left[\left\langle m_{2},\right\rangle\right]$ given by sending $\left[F_{n}\right]$ to $\left[\left\langle m_{2}, F_{n}\right\rangle\right]$. The square of $\left[\left\langle m_{2}, \quad\right\rangle\right]$ is zero precisely because $\left[\left\langle m_{2},\left\langle m_{2}, F_{n}\right\rangle\right\rangle\right]$ equals $\left[-\left\langle m_{3},\left\langle m_{1}, F_{n}\right\rangle\right\rangle-\left\langle m_{1},\left\langle m_{3}, F_{n}\right\rangle\right\rangle\right]$. The first term $\left\langle m_{3}\left\langle m_{1}, F_{n}\right\rangle\right\rangle$ is zero because $\left\langle m_{1}, F_{n}\right\rangle=0$. The second term is the coboundary of $\left\langle m_{3}, F_{n}\right\rangle$, so its class with respect to $\left\langle m_{1},\right\rangle$ is zero.

There is an algebra splitting from $R$ to $E_{1}$ given by sending $f_{n}$ to $\left[\lambda_{2} f_{n} p_{1}\right]$, so $E_{1} \approx R$ as graded commutative algebras. Furthermore, since $\mathrm{H}_{m_{1}}\left(m_{2}\right)=\widehat{m_{2}}$ and $\mathrm{H}_{l_{1}}\left(l_{2}\right)=\widehat{l_{2}}$, it follows that $\left[\left\langle m_{2}, \quad\right\rangle\right]=\left\langle\widehat{m_{2}}, \quad\right\rangle$. Therefore, $E_{1}$ and $R$ are isomorphic as differential graded commutative algebras and have the same cohomology.

Constructing homotopy Lie-Rinehart resolutions for a Lie-Rinehart pair is no easy task. In an upcoming paper, we construct a homotopy Lie-Rinehart resolu- 
tion for the Lie-Rinehart pair $\left(A / \mathcal{I}, \mathcal{I} / \mathcal{I}^{2}\right)$ which arises in the BFV formulation of classical BRST cohomology.

\section{References}

[BF83] I. Batalin and E. Fradkin. A generalized canonical formalism and quantization of reducible gauge theories. Physics Letters B, 122:157$164,1983$.

[BRS75] C. Becchi, A. Rouet, and R. Stora. Renormalization of the abelian Higgs-Kibble model. Commun. Math. Phys., 42:127, 1975.

[BV77] I. Batalin and G. Vilkovisky. Relativistic S-matrix of dynamical systems with boson and fermion constraints. Physics Letters B, 69:309312, 1977.

[BV83] I. Batalin and G. Vilkovisky. Quantization of gauge theories with linearly dependent generators. Physics Review D, 28:2567-2582, 1983.

[BV85] I. Batalin and G. Vilkovisky. Existence theorem for gauge algebra. Journal of Mathematical Physics, 26:172-184, 1985.

[CE48] C. Chevalley and S. Eilenberg. Cohomology theory of Lie groups and Lie algebras. Trans. Amer. Math. Soc., 63:85-124, 1948.

[dSW] A.C. do Silva, , and A. Weinstein. Lectures on geometric models for noncommutative algebras. UC-Berkeley.

[FHST89] J. Fisch, M. Henneaux, J. Stasheff, and C. Teitelboim. Existence, uniqueness and cohomology of the classical BRST charge with ghosts of ghosts. Commun. Math. Phys., 120:379, 1989.

[FV75] E. Fradkin and G. Vilkovisky. Quantization of relativistic systems with constraints. Physics Letters B, 55:224-226, 1975.

[GL89] V.K.A.M. Gugenheim and L. Lambe. Applications of perturbation theory to differential homological algebra i. Illinois Journal of Mathematics, 33, 1989.

[GLS91] V.K.A.M. Gugenheim, L. Lambe, and J.D. Stasheff. Perturbation theory in differential homological algebra ii. Illinois Journal of Mathematics, 35(3):357-373, 1991.

[Her72] G. C. Herz. Cotriples and the Chevalley-Eilenberg cohomology of Lie algebra. PhD thesis, Lehigh University, 1972.

[HT92] M. Henneaux and C. Teitelboim. Quantization of Gauge Systems. Princeton University Press, 1992.

[Kim92a] T. Kimura. Classical BRST cohomology and second class constraints. XIX International Colloquium on Group Theoretical Methods in Physics in Salamanca, Spain, July 1992.

[Kim92b] T. Kimura. Prequantum BRST cohomology. Contemporary Mathematics, 132:439-457, 1992.

[Kim93] T. Kimura. Generalized classical BRST cohomology and reduction of Poisson manifolds. Commun. Math. Phys., 151:155-182, 1993. 
[KS87] B. Kostant and S. Sternberg. Symplectic reduction, BRS cohomology, and infinite-dimensional clifford algebras. Annals of Physics, 176:49113, 1987.

[Lad99] T. Lada. Commutators of a infinity structures. Conference Proceedings of Higher Homotopy Structures in Topology and Mathematical Physics, Contemporary Mathematics, 227:227-233, 1999.

[LM95] T. Lada and M. Markl. Strongly homotopy lie algebras. Commun. in Alg., 23(6):2147-2161, 1995.

[LS93] T. Lada and J. D. Stasheff. Introduction to sh Lie algebras for physicists. International J. of Theor. Phys., 32:1087-1103, 1993.

[Pal61] R. S. Palais. The cohomology of Lie rings. In Proceedings of the Sympososium in Pure Mathematic, Vol. III., pages 130-137. American Mathematical Society, 1961.

[Rin63] G. Rinehart. Differential forms for general commutative algebras. Trans. Amer. Math. Soc., 108:195-222, 1963.

[SS] M. Schlessinger and J. D. Stasheff. Deformation theory and rational homotopy type. to appear in Publ. Math. IHES.

[Sta88] J. D. Stasheff. Constrained Poisson algebras and strong homotopy representations. Bull. Amer. Math. Soc., pages 287-290, 1988.

[Sta92] J. D. Stasheff. Homological (ghost) approach to constrained hamiltonian systems. Contemp. Math., 132:595, 1992.

[Sta96] J. D. Stasheff. Homological reduction of constrained Poisson algebras I: the regular case. Journal of Differential Geometry, ?:?, 1996.

[Sul77] D. Sullivan. Infinitesimal computations in topology. Publ. IHES, 47:269-331, 1977.

[Tat57] J. Tate. Homology of Noetherian rings and local rings. Ill. J. Math., 1:14-27, 1957.

[Tyu75] I. Tyutin. Gauge invariance in field theory and statistical physics in operator formulation (in Russian). Lebedev Physics Inst., 39, 1975.

[Wei94] C. Weibel. An introduction to homological algebra. Cambridge studies in advanced mathematics. Cambridge University Press, 1994.

This article may be accessed via WWW at http://www.rmi.acnet.ge/hha/ or by anonymous ftp at

ftp://ftp.rmi.acnet.ge/pub/hha/volumes/2001/n7/n7.(dvi,ps,dvi.gz,ps.gz)

Lars Kjeseth 1kjeseth@elcamino.cc.ca.us

Mathematical Sciences Division

El Camino College

U.S.A.

Torrance

16007 Crenshaw Blvd, Torrance, CA 90506 